\title{
SPECTRAL APPROXIMATIONS BY THE HDG METHOD
}

\author{
J. GOPALAKRISHNAN, F. LI, N.-C. NGUYEN, AND J. PERAIRE
}

\begin{abstract}
We consider the numerical approximation of the spectrum of a second-order elliptic eigenvalue problem by the hybridizable discontinuous Galerkin (HDG) method. We show for problems with smooth eigenfunctions that the approximate eigenvalues and eigenfunctions converge at the rate $2 k+1$ and $k+1$, respectively. Here $k$ is the degree of the polynomials used to approximate the solution, its flux, and the numerical traces. Our numerical studies show that a Rayleigh quotient-like formula applied to certain locally postprocessed approximations can yield eigenvalues that converge faster at the rate $2 k+2$ for the HDG method as well as for the Brezzi-Douglas-Marini (BDM) method. We also derive and study a condensed nonlinear eigenproblem for the numerical traces obtained by eliminating all the other variables.
\end{abstract}

\section{INTRODUCTION}

We study the HDG (hybridized discontinuous Galerkin) approximation to the following eigenproblem: Find eigenvalues $\lambda$ in $\mathbb{R}$ and corresponding nontrivial eigenfunctions $u$ satisfying

$$
-\nabla \cdot(\alpha \vec{\nabla} u)=\lambda u \quad \text { in } \Omega, \quad u=0 \quad \text { on } \partial \Omega .
$$

Assumptions are placed on $\alpha$ and $\Omega$ in Section 2. Several HDG discretizations were introduced in 8 to discretize corresponding source problems. The purpose of this paper is to study the application of one such method to eigenproblems. The particular HDG method considered here (referred to as the H-LDG method in 8 , but simply as the "HDG method" in this paper) is chosen for our study because we now have a fairly complete theoretical understanding of its application to the source problem.

Two well-known advantages of the HDG method, when applied to source problems, are reduced system size and flexible stabilization. The latter arises due to a transparent stabilization mechanism in the definition of numerical flux. It allows one to use, for example, polynomials of the same degree $k$ to approximate the solution $u$ and components of its flux $\vec{q}=-\alpha \vec{\nabla} u$. While use of these spaces would have resulted in an unstable mixed method, the resulting HDG method is stable, and provides optimal order approximations for all variables. To discuss the former, namely the advantage of reduced system size, recall the process of static condensation, which, for source problems, removes all interior variables to yield a

Received by the editor July 3, 2012 and, in revised form, July 11, 2013.

2010 Mathematics Subject Classification. Primary 65N12, 65N25, 65N30.

Key words and phrases. HDG, nonlinear, eigenvalue, eigenfunction, BDM, postprocessing, condensation, hybridization, pollution, perturbation.

This work was partially supported by NSF through grants DMS-1211635, DMS-1318916, and the CAREER award DMS-0847241, by an Alfred P. Sloan Research Fellowship, and by AFOSR under grant FA9550-12-0357. 
"condensed" system for inter-element variables. The HDG condensed system, when compared to condensed systems from other DG methods, is attractive because of its smaller size and favorable sparsity patterns. One of the questions we address in this paper is whether such condensed systems are useful in eigenproblems. Note that the condensation process, by reducing the system size, also reduces the size of the spectrum. How much of the spectrum can be approximated despite this reduction is a question answered in Section 5. There, we also derive the nonlinear equation that needs to be solved in the reduced dimensions to compute the spectral approximations.

Apart from these results on the condensed eigenproblem, we prove convergence results for the HDG eigenproblem in Sections 3 and 4 . We show that the approximate eigenvalues obtained by the HDG method exhibit no spectral pollution. They converge to the exact eigenvalues at the rate $O\left(h^{2 k+1}\right)$, under suitable regularity assumptions, if we use polynomials of degree at most $k$ for all the HDG variables. We also show, under similar assumptions, that the gap between the corresponding discrete eigenspace and the exact eigenspace in $L^{2}(\Omega)$ converges at $O\left(h^{k+1}\right)$. Roughly speaking, this shows that the rate of convergence of eigenfunctions in $L^{2}(\Omega)$ is optimal for the HDG method.

These results compare favorably with many other DG eigenvalue approximations [2, 13. The unified presentation of 2] facilitates comparison. They show that many traditional Hermitian DG methods approximate eigenvalues at the rate $O\left(h^{2 k}\right)$. For non-Hermitian DG methods, they find that the eigenvalue convergence rate is even poorer, in general no better than $O\left(h^{k}\right)$. The HDG method, which can be considered to fall in the Hermitian class, yields eigenvalues that converge faster, when compared to both the Hermitian and the non-Hermitian DG methods considered in [2]. However, let us note that the convergence rate of HDG eigenvalues (or, for that matter, any DG eigenvalues) do not compare favorably with the $O\left(h^{2 k+2}\right)$ convergence rate of the mixed hybridized Raviart-Thomas (HRT) method [5, 9, 16, Our analysis also points to other differences when comparing the HDG and HRT eigenproblems. For example, comparing Theorem [5.3 below with [9, Theorem 3.2], we note that the extent of the spectrum recovered by the condensed system may be shorter (up to $O(1 / h)$ ) for the HDG method in comparison to the HRT method (which is up to $O\left(1 / h^{2}\right)$ ).

The method of convergence analysis in this paper is motivated by the many early works that developed abstract approaches to analyze approximation of eigenproblems [6, 11, 16, 17], and in particular, the application of the abstract theory to DG methods in [2]. The critical new tool that helps push the analysis forward in the HDG case, and yield better convergence rates than [2], is the projection operator of [10. The projection allows our analysis of HDG eigenvalue errors to proceed along the lines of similar analyses for mixed methods [5]. A few important differences arise due to the fact that the HDG projection possesses only a weak analogue of a well-known commutativity property. The analysis in the second aspect of this study, involving the condensed system, is motivated by our previous such analysis 9] for the HRT method.

Reports of extensive numerical experiments are provided in Section 6. Of particular interest is a local and inexpensive postprocessing presented there. The postprocessed eigenvalues seem to converge at $O\left(h^{2 k+2}\right)$-rate thus making the HDG 
method competitive with the mixed method. The intuition behind the construction of this postprocessing is inherited from our previous experience with the mixed method [9], where we proved that the postprocessed eigenfunctions have better convergence rates. However, the argument used there to provide a rigorous proof does not seem to extend to the HDG method.

In the next section, we introduce the HDG eigenproblem and essential notations used throughout the paper. In Section 3 , we show that there is no pollution of the spectrum when approximating it by the HDG method. This result is improved in Section 4, where we establish (in Theorem 4.1) the convergence rate of HDG eigenvalues. Section 5 investigates the condensation (or hybridization) of the HDG eigenproblem to obtain a smaller condensed nonlinear eigenproblem for the interface variable, otherwise known as the numerical trace. The condensed nonlinear eigenproblem is given in Theorem 5.3 and a closely related linear eigenproblem is investigated in Theorem 5.4 .

We conclude in Section 6 with the results of our numerical studies and a brief discussion of the eigenproblem using mixed-degree polynomial spaces.

\section{The HDG SOURCE AND EIGENVAlue PRoblems}

Consider the Dirichlet boundary value problem (rewritten as a first order system) of finding $\vec{q}^{f} \in H(\operatorname{div}, \Omega)$ and $u^{f} \in L^{2}(\Omega)$, given any "source" $f$ in $L^{2}(\Omega)$, such that

$$
\begin{aligned}
\vec{q}^{f}+\alpha \vec{\nabla} u^{f} & =0 & & \text { on } \Omega, \\
\nabla \cdot \vec{q}^{f} & =f & & \text { on } \Omega, \\
u^{f} & =0 & & \text { on } \partial \Omega .
\end{aligned}
$$

All functions, unless explicitly stated otherwise, are real-valued in this paper. Throughout, $\Omega \subset \mathbb{R}^{n}$ is a polyhedral domain $(n \geq 2), \alpha: \Omega \rightarrow \mathbb{R}^{n \times n}$ denotes a variable matrix valued coefficient, which we assume to be symmetric and positive definite at all points in $\Omega$. We assume that there is a fixed constant that bounds the norms of $\alpha$ and $c=\alpha^{-1}$ for all $x \in \Omega$, on which dependence is not tracked in the estimates of this paper. To facilitate our analysis, we introduce notation for the "solution operator" $T: L^{2}(\Omega) \rightarrow L^{2}(\Omega)$, which is defined simply by

$$
T f=u^{f} \text {. }
$$

It is well known that $T$ is compact and self-adjoint. Its spectrum, denoted by $\sigma(T)$, consists of isolated points on the positive real line accumulating at zero. Clearly, there is a one-to-one correspondence between the eigenvalues of (11) and those of $T$. Indeed, $\mu$ is an eigenvalue of $T$ if and only if $\mu=1 / \lambda$ for some $\lambda$ satisfying (1).

2.1. The source problem. The HDG method provides an approximation $T_{h}$ to $T$. To understand this approximation, we first describe the HDG source problem and introduce known results we shall use later. Afterward, we will present the HDG eigenvalue problem.

The HDG method defines a scalar approximation $u_{h}$ to $u$ and a vector approximation $\vec{q}_{h}$ to $\vec{q}$ in the following spaces, respectively:

$$
\begin{aligned}
W_{h} & =\left\{w: \text { for every mesh element } K,\left.w\right|_{K} \in P_{k}(K)\right\}, \\
V_{h} & =\left\{\vec{v}: \text { for every mesh element } K,\left.\vec{v}\right|_{K} \in P_{k}(K)^{n}\right\} .
\end{aligned}
$$


Note that functions in these spaces need not be continuous across element interfaces. Above and elsewhere, we use $P_{k}(D)$ to denote the space of polynomials of degree at most $k \geq 0$ on some domain $D$. The subscript $h$ denotes the mesh size defined as the maximum of the diameters of all mesh elements.

For any (scalar or vector) function $q$ in $V_{h}$ or $W_{h}$, the trace $\left.q\right|_{F}$ is, in general, a double-valued function on any interior mesh face $F=\partial K^{+} \cap \partial K^{-}$shared by the mesh elements $K^{+}$and $K^{-}$. Its two branches, denoted by $[q]_{K^{+}}$and $[q]_{K^{-}}$, are defined by $[q]_{K^{ \pm}}(\vec{x})=\lim _{\epsilon \downarrow 0} q\left(\vec{x}-\epsilon[\vec{n}]_{K^{ \pm}}\right)$for all $\vec{x}$ in $F$. Here and elsewhere, $\vec{n}$ denotes the double-valued function of unit normals on the element interfaces: on a face $F \subseteq \partial K$, its branch $[\vec{n}]_{K}$ equals the unit normal on $\partial K$ pointing outward from $K$. For functions $u$ and $v$ in $L^{2}(D)$, we write $(u, v)_{D}=\int_{D} u v d x$ whenever $D$ is a domain of $\mathbb{R}^{n}$, and $\langle u, v\rangle_{D}=\int_{D} u v d x$ whenever $D$ is a domain of $\mathbb{R}^{n-1}$. To simplify the notation, define

$$
(v, w)_{\mathscr{T}_{h}}=\sum_{K \in \mathscr{T}_{h}}(v, w)_{K} \quad \text { and } \quad\langle v, w\rangle_{\partial \mathscr{T}_{h}}=\sum_{K \in \mathscr{T}_{h}}\langle v, w\rangle_{\partial K},
$$

where in the latter, we understand that for double-valued $v$ and $w$, the integral $\langle v, w\rangle_{\partial K}$ is computed using the branches $[v]_{K}$ and $[w]_{K}$ from $K$. For vector functions $\vec{v}$ and $\vec{w}$, the notations are similarly defined with the integrand being the dot product $\vec{v} \cdot \vec{w}$.

In addition to the spaces $V_{h}$ and $W_{h}$ introduced above, our method also uses one other discrete space $M_{h}$, consisting of functions defined on the mesh faces (or mesh edges if $n=2$ ), namely

(6) $M_{h}=\left\{\mu\right.$ : for every mesh face $F,\left.\mu\right|_{F} \in P_{k}(F)$, and if $\left.F \subseteq \partial \Omega,\left.\mu\right|_{F}=0\right\}$.

The HDG method defines the approximate solution $u_{h}^{f}$, the approximate flux $\vec{q}_{h}^{f}$, and the numerical trace $\eta_{h}^{f}$, as the functions in $W_{h}, V_{h}$, and $M_{h}$, respectively, satisfying

$$
\begin{aligned}
\left(c \vec{q}_{h}^{f}, \vec{r}\right)_{\mathscr{T}_{h}}-\left(u_{h}^{f}, \nabla \cdot \vec{r}\right)_{\mathscr{T}_{h}}+\left\langle\eta_{h}^{f}, \vec{r} \cdot \vec{n}\right\rangle_{\partial \mathscr{T}_{h}} & =0, & & \text { for all } \vec{r} \in V_{h}, \\
-\left(\vec{q}_{h}^{f}, \vec{\nabla} w\right)_{\mathscr{T}_{h}}+\left\langle\widehat{q}_{h}^{f} \cdot \vec{n}, w\right\rangle_{\partial \mathscr{T}_{h}} & =(f, w)_{\mathscr{T}_{h}} & & \text { for all } w \in W_{h}, \\
\left\langle\mu, \widehat{q}_{h}^{f} \cdot \vec{n}\right\rangle_{\partial \mathscr{T}_{h}} & =0 & & \text { for all } \mu \in M_{h},
\end{aligned}
$$

where $\widehat{q}_{h}^{f}$ is a double-valued vector function on mesh interfaces defined by

$$
\widehat{q}_{h}^{f}=\vec{q}_{h}^{f}+\tau\left(u_{h}^{f}-\eta_{h}^{f}\right) \vec{n} .
$$

Note that this defines all branches, i.e., on the boundary $\partial K$ of every mesh element $K$, the value of the branch of $\widehat{q}_{h}^{f}$ from $K$ is $\left[\widehat{q}_{h}^{f}\right]_{K}=\left[\vec{q}_{h}^{f}\right]_{K}+[\tau]_{K}\left(\left[u_{h}^{f}\right]_{K}-\eta_{h}^{f}\right)[\vec{n}]_{K}$. Here $\tau$ is a nonnegative penalty function, which is also double-valued on the element interfaces and $[\tau]_{K}$ above denotes the branch of $\tau$-values from $K$. For simplicity, we assume that any branch of $\tau$ is a constant function on each mesh edge. It is proved in [8] that the system (7) is uniquely solvable if $[\tau]_{K}$ is positive on at least one face of $K$ for every element $K$. This unique solvability result is assumed throughout this paper. Given any $f$ in $L^{2}(\Omega)$, the component $u_{h}^{f}$ of the unique solution of (7) is used to define the discrete version of the operator $T$ in (3), namely

$$
T_{h} f=u_{h}^{f} .
$$


We will need a projection $\Pi_{h}(\vec{q}, u)$, into the product space $V_{h} \times W_{h}$, originally designed in 10. Its domain is a subspace of $H(\operatorname{div}, \Omega) \times L^{2}(\Omega)$ consisting of sufficiently regular functions, e.g., $H(\operatorname{div}, \Omega) \cap H^{s}(\Omega)^{n} \times H^{s}(\Omega)$ for $s>1 / 2$. When its components need to be identified, we also write $\Pi_{h}(\vec{q}, u)$ as $\left(\Pi_{h}^{V} \vec{q}, \Pi_{h}^{W} u\right)$ where $\Pi_{h}^{V} \vec{q}$ and $\Pi_{h}^{W} u$ are the components of the projection in $V_{h}$ and $W_{h}$, respectively. (Despite this notation, note that $\Pi_{h}^{V} \vec{q}$ depends not just on $\vec{q}$, but rather on both $\vec{q}$ and $u$. The same applies for $\Pi_{h}^{W} u$.) The components are defined by

$$
\begin{aligned}
\left(\Pi_{h}^{V} \vec{q}, \vec{r}\right)_{K} & =(\vec{q}, \vec{r})_{K} & & \text { for all } \vec{r} \in P_{k-1}(K)^{n}, \\
\left(\Pi_{h}^{W} u, w\right)_{K} & =(u, w)_{K} & & \text { for all } w \in P_{k-1}(K), \\
\left\langle\Pi_{h}^{V} \vec{q} \cdot \vec{n}+\tau \Pi_{h}^{W} u, \mu\right\rangle_{F} & =\langle\vec{q} \cdot \vec{n}+\tau u, \mu\rangle_{F} & & \text { for all } \mu \in P_{k}(F),
\end{aligned}
$$

for all faces $F$ of the simplex $K$. Let $s_{u}, s_{q} \in(1 / 2, k+1]$. We recall the following approximation property, proved in [10, Theorem 2.1] for integer values of $s_{u}, s_{q}$, and extended to remaining values of $s_{u}, s_{q} \in(1 / 2, k+1]$ in [7]:

For all $(\vec{q}, u) \in H(\operatorname{div}, \Omega) \cap H^{s_{q}}(\Omega)^{n} \times H^{s_{u}}(\Omega)$,

$$
\begin{aligned}
\left\|\Pi_{h}^{V} \vec{q}-\vec{q}\right\|_{L^{2}(K)} & \leq C h_{K}^{s_{q}}|\vec{q}|_{H^{s_{q}(K)}}+C h_{K}^{s_{u}} \tau_{K}^{*}|u|_{H^{s_{u}}(K)} \\
\left\|\Pi_{h}^{W} u-u\right\|_{L^{2}(K)} & \leq C h_{K}^{s_{u}}|u|_{H^{s_{u}}(K)}+C \frac{h_{K}^{s_{q}}}{\tau_{K}^{\max }}|\vec{q}|_{H^{s_{q}(K)}} .
\end{aligned}
$$

Above and throughout we use $C$ to denote a generic constant independent of the mesh element sizes and the stabilization parameter $\tau$. The notations appearing above are defined as follows, letting $F_{\max }$ denote the face of $K$ where $\left.\tau\right|_{\partial K}$ is maximum:

$$
\tau_{K}^{\max }=\left.\max \tau\right|_{\partial K}, \quad \tau_{K}^{*}=\left.\max \tau\right|_{\partial K \backslash F_{\max }}, \quad h_{K}=\operatorname{diam}(K), \quad h=\max _{K \in T h} h_{K} .
$$

The following error estimate is known [7, 10].

Theorem 2.1 (see [7]). Let the exact solution $\left(\vec{q}^{f}, u^{f}\right)$ of (2) be in $H(\operatorname{div}, \Omega) \cap$ $H^{s}(\Omega)^{n} \times H^{s}(\Omega)$ for some $s>1 / 2$. Then,

$$
\begin{aligned}
\left\|u-u_{h}^{f}\right\|_{\mathscr{T}_{h}} & \leq C\left\|u-\Pi_{h}^{W} u\right\|_{\mathscr{T}_{h}}+b_{\tau} C\left\|\vec{q}-\Pi_{h}^{V} \vec{q}\right\|_{c}, \\
\left\|\vec{q}-\vec{q}_{h}^{f}\right\|_{c} & \leq 2\left\|\vec{q}-\Pi_{h}^{V} \vec{q}\right\|_{c},
\end{aligned}
$$

where $b_{\tau}=\max \left\{1+h_{K} \tau_{K}^{*}+h_{K} / \tau_{K}^{\max }: K \in \mathscr{T}_{h}\right\}$, and $\|\vec{q}\|_{c}=(c \vec{q}, \vec{q})_{\partial \mathscr{T}_{h}}^{1 / 2}$ with $c=$ $\alpha^{-1}$.

2.2. The eigenproblem. The HDG discretization of the eigenproblem (1) defines an approximation to the eigenfunction $u_{h} \in W_{h}$, an approximation to the eigenflux $\vec{q}_{h} \in V_{h}$, and an approximation to the eigenfunction trace $\eta_{h} \in M_{h}$, as a nontrivial set of functions satisfying

$$
\begin{aligned}
\left(c \vec{q}_{h}, \vec{r}\right)_{\mathscr{T}_{h}}-\left(u_{h}, \nabla \cdot \vec{r}\right)_{\mathscr{T}_{h}}+\left\langle\eta_{h}, \vec{r} \cdot \vec{n}\right\rangle_{\partial \mathscr{T}_{h}} & =0, & & \text { for all } \vec{r} \in V_{h}, \\
-\left(\vec{q}_{h}, \vec{\nabla} w\right)_{\mathscr{T}_{h}}+\left\langle\widehat{q}_{h} \cdot \vec{n}, w\right\rangle_{\partial \mathscr{T}_{h}} & =\lambda_{h}\left(u_{h}, w\right)_{\mathscr{T}_{h}} & & \text { for all } w \in W_{h}, \\
\left\langle\mu, \widehat{q_{h}} \cdot \vec{n}\right\rangle_{\partial \mathscr{T}_{h}} & =0 & & \text { for all } \mu \in M_{h},
\end{aligned}
$$

where $\widehat{q}_{h}$ is defined by $\widehat{q}_{h}=\vec{q}_{h}+\tau\left(u_{h}-\eta_{h}\right) \vec{n}$, cf. (8). Here, $\lambda_{h} \in \mathbb{R}$ is the corresponding discrete eigenvalue.

The unique solvability of the source problem (17) implies that $\lambda_{h}$ is nonzero. One can easily verify that $\mu_{h}$ is an eigenvalue of $T_{h}$ if and only if $\mu_{h}=1 / \lambda_{h}$ for some $\lambda_{h}$ solving (14). Moreover, $\lambda_{h}$ is positive as can be concluded from the next lemma. 
Lemma 2.2. $T_{h}$ is self-adjoint and positive definite in $L^{2}(\Omega)$.

Proof. To show that $\left(f, T_{h} g\right)=\left(T_{h} f, g\right)$, where $(\cdot, \cdot)$ denotes the $L^{2}(\Omega)$-inner product, we calculate as follows:

$$
\begin{aligned}
& \left(f, T_{h} g\right)=-\left(\vec{q}_{h}^{f}, \vec{\nabla} u_{h}^{g}\right)_{\mathscr{T}_{h}}+\left\langle\widehat{q}_{h}^{f} \cdot \vec{n}, u_{h}^{g}\right\rangle_{\partial \mathscr{T}_{h}}, \\
& =\left(\nabla \cdot \vec{q}_{h}^{f}, u_{h}^{g}\right)_{\mathscr{T}_{h}}+\left\langle\left(\widehat{q}_{h}^{f}-\vec{q}_{h}^{f}\right) \cdot \vec{n}, u_{h}^{g}\right\rangle_{\partial \mathscr{T}_{h}} \\
& =\left(c \vec{q}_{h}^{g}, \vec{q}_{h}^{f}\right)_{\mathscr{T}_{h}}+\left\langle\eta_{h}^{g}, \vec{q}_{h}^{f} \cdot \vec{n}\right\rangle_{\partial \mathscr{T}_{h}}+\left\langle\left(\widehat{q}_{h}^{f}-\vec{q}_{h}^{f}\right) \cdot \vec{n}, u_{h}^{g}\right\rangle_{\partial \mathscr{T}_{h}} \\
& =\left(c \vec{q}_{h}^{g}, \vec{q}_{h}^{f}\right)_{\mathscr{T}_{h}}-\left\langle\vec{q}_{h}^{f} \cdot \vec{n}, u_{h}^{g}-\eta_{h}^{g}\right\rangle_{\partial \mathscr{T}_{h}}+\left\langle\widehat{q}_{h}^{f} \cdot \vec{n}, u_{h}^{g}-\eta_{h}^{g}\right\rangle_{\partial \mathscr{T}_{h}} \\
& =\left(c \vec{q}_{h}^{g}, \vec{q}_{h}^{f}\right)_{\mathscr{T}_{h}}+\left\langle\tau\left(u_{h}^{f}-\eta_{h}^{f}\right),\left(u_{h}^{g}-\eta_{h}^{g}\right)\right\rangle_{\partial \mathscr{T}_{h}} \\
& \text { by (7b) with } w=T_{h} g=u_{h}^{g} \\
& \text { by integration by parts } \\
& \text { by (7a) with } \vec{r}=\vec{q}_{h}^{f} \\
& \text { by (7c) with } \mu=\eta_{h}^{g} \\
& \text { by (8). }
\end{aligned}
$$

The last expression is symmetric in $f$ and $g$ and is nonnegative if $f=g$. This proves that $T_{h}$ is self-adjoint and positive semidefinite in $L^{2}(\Omega)$. As already noted previously, zero is not an eigenvalue of $T_{h}$, hence $T_{h}$ is positive definite.

\section{Convergence of the spectrum}

The convergence of the discrete eigenvalues to the exact ones is proved by establishing convergence of $T_{h}$ to $T$ in operator norm. (Recall that $T$ and $T_{h}$ are defined in (3) and (9), respectively.) Such operator convergence was used as the basis for the early analyses of spectral approximations using conforming methods [6, 16, 17. It has also been used to analyze approximations of eigenvalue problems using older discontinuous Galerkin methods (like the interior penalty method) [2]. To apply this technique to the HDG eigenproblem, we need the following basic result.

Theorem 3.1 (Operator convergence). Suppose there is an $s>1 / 2$ such that any solution $\left(\vec{q}^{f}, u^{f}\right)$ of (2) satisfies

$$
\left\|\vec{q}^{f}\right\|_{H^{s}(\Omega)}+\left\|u^{f}\right\|_{H^{s}(\Omega)} \leq C\|f\|_{L^{2}(\Omega)},
$$

for all $f \in L^{2}(\Omega)$. Then

$$
\left\|T-T_{h}\right\| \leq c_{\tau} h^{\min (s, k+1)}
$$

where $\|\cdot\|$ denotes the $L^{2}(\Omega)$-operator norm and $c_{\tau}=C \max \left\{1+h_{K}^{2}\left(\tau_{K}^{*}\right)^{4}+\right.$ $\left.\left(\tau_{K}^{\max }\right)^{-2}: K \in \mathscr{T}_{h}\right\}^{1 / 2}$.

Proof. The convergence results for the HDG source problem imply

$$
\begin{array}{rlrl}
\left\|T f-T_{h} f\right\|_{L^{2}(\Omega)} & \leq C\left\|u-\Pi_{h}^{W} u\right\|_{\mathscr{T}_{h}}+b_{\tau} C\left\|\vec{q}-\Pi_{h}^{V} \vec{q}\right\|_{c}, & & \text { by Theorem 2.1] eq. (12) }, \\
& \leq C c_{\tau} h^{\min (s, k+1)}\left(|q|_{H^{s}(\Omega)}+|u|_{H^{s}(\Omega)}\right), & \text { by (11). }
\end{array}
$$

Hence the result follows from (15).

Note that assumption (15) is a regularity assumption that holds, for example, when $s \equiv 1$ and $\Omega$ is a polygonal domain (with no cracks) in $\mathbb{R}^{2}$.

By virtue of Theorem 3.1 and the well-known consequences of operator convergence 1, 12, 17, we conclude that the spectrum of $T_{h}$ approximates that of $T$, i.e., there is no "pollution" of the spectrum when it is approximated by the HDG method. To formulate the statement of this approximation precisely in a form we can use later, let us recall some standard terminology. The "gap" between two subspaces $X$ and $Y$ of $L^{2}(\Omega)$ is defined by

$$
\delta(X, Y)=\sup _{x \in X} \frac{\operatorname{dist}(x, Y)}{\|x\|_{L^{2}(\Omega)}}=\sup _{y \in Y} \frac{\operatorname{dist}(y, X)}{\|y\|_{L^{2}(\Omega)}} .
$$


Now, suppose $\mu$ is an eigenvalue of $T$ of multiplicity $m$ and let $\Gamma$ be a positively oriented circle, contained in the resolvent set of $T$, centered at $\mu$, and enclosing no other eigenvalue of $T$. Define two operators, $E_{h}^{\Gamma}$ and $E^{\Gamma}$, both on $L^{2}(\Omega)$, by the following integrals over $\Gamma$ in the complex plane:

$$
E_{h}^{\Gamma}=\frac{1}{2 \pi \hat{\imath}} \oint_{\Gamma}\left(z-T_{h}\right)^{-1} d z, \quad E^{\Gamma}=\frac{1}{2 \pi \hat{\imath}} \oint_{\Gamma}(z-T)^{-1} d z .
$$

Hereon, we omit the superscript $\Gamma$ in $E_{h}^{\Gamma}$ and $E^{\Gamma}$ as $\Gamma$ will always be taken as stated above. A well-known result is that $E$ is a projection onto the eigenspace of $T$ corresponding to the eigenvalue $\mu$. The discrete analogue of this result for $E_{h}$ appears in results collected below. The collection summarizes a few consequences of operator convergence. The arguments proving these consequences are standard [2, 16, 17, and since they apply to the HDG context with few modifications, we shall not repeat them. We use $R(A)$ to denote the range of any operator $A$ and $C_{\tau}$ to denote generic constants independent of $h$, but dependent on $\tau$.

Corollary 3.2 (Convergence of eigenvalues and eigenfunctions). Let $\mu$ and $\Gamma$ be as above and let the assumption of Theorem 3.1 hold for some $s>1 / 2$. Then, there exists $h_{\Gamma}>0$ such that for all $h<h_{\Gamma}$ the following statements hold:

(1) There are exactly $m$ eigenvalues of $T_{h}$ within $\Gamma$, which we count according to multiplicity and enumerate as $\mu_{h, 1}, \mu_{h, 2}, \ldots, \mu_{h, m}$.

(2) The operator $E_{h}$ is a projection onto the span of the eigenfunctions of $T_{h}$ corresponding to all the eigenvalues $\mu_{h, j}$ for $j=1, \ldots, m$.

(3) The operator $E_{h}$ converges to $E$ as $h \rightarrow 0$ and

(4) The exact and discrete eigenspaces (of $\mu$ and $\left\{\mu_{h, j}\right\}_{j=1}^{m}$, respectively) are $R(E)$ and $R\left(E_{h}\right)$, respectively. The discrete eigenspaces converge in the sense that

$$
\delta\left(R(E), R\left(E_{h}\right)\right) \leq C_{\tau} h^{\min (s, k+1)} .
$$

(5) If, in addition, the eigenfunctions of $\mu$ have a higher regularity index, i.e., if

$$
\left\|\vec{q}^{f}\right\|_{H^{s \mu}(\Omega)}+\left\|u^{f}\right\|_{H^{s \mu}(\Omega)} \leq C\|f\|_{L^{2}(\Omega)}, \quad \forall f \in R(E),
$$

with $s_{\mu} \geq s$, then (17) can be refined to

$$
\left\|\left.\left(E-E_{h}\right)\right|_{R(E)}\right\| \leq C\left\|\left.\left(T-T_{h}\right)\right|_{R(E)}\right\| \leq C_{\tau} h^{\min \left(s_{\mu}, k+1\right)}
$$

and, consequently, (18) can be revised to $\delta\left(R(E), R\left(E_{h}\right)\right) \leq C_{\tau} h^{\min \left(s_{\mu}, k+1\right)}$.

\section{RATE OF CONVERGENCE OF EIGENVALUES}

In this section, we prove that under favorable regularity conditions, the HDG eigenvalues converge at the rate $O\left(h^{2 k+1}\right)$ when we use polynomials of degree at most $k \geq 0$ for all variables. To do so, we begin with the setting of Corollary 3.2 and refine a few estimates through a duality argument. Accordingly, we keep the same notations as in Corollary 3.2, and tacitly assume throughout this section that the assumptions in the corollary hold. In particular, recall that $R(E)$ is the eigenspace of $T$ corresponding to an eigenvalue $\mu$ and $\mu_{h, j}$ are the discrete eigenvalues near $\mu$. 
Theorem 4.1. Suppose there is an $s_{\mu}>1 / 2$ such that

$$
\left\|\vec{q}^{f}\right\|_{H^{s^{\mu}}(\Omega)}+\left\|u^{f}\right\|_{H^{s_{\mu}+1}(\Omega)} \leq C\|f\|_{L^{2}(\Omega)}, \quad \forall f \in R(E) .
$$

Then there is an $h_{\mu}>0$ such that for all $h<h_{\mu}$,

$$
\left|\mu-\mu_{h, j}\right| \leq C h^{\min \left(s_{\mu}, k+1\right)+\min \left(s_{\mu}, k\right)} .
$$

This is the main result of this section, and in the remainder of this section, we prove it. As we shall see, we are able to apply the needed duality techniques for this proof, thanks to properties of the projection operator $\Pi_{h}(\vec{q}, u)$ of [10], recalled in Subsection 2.1. Given any eigenfunction $e \in R(E)$, let us denote its corresponding flux by

$$
\overrightarrow{q_{e}}=-\alpha \vec{\nabla} e .
$$

Then $\Pi_{h}^{W} e$ is the component of $\Pi_{h}\left(\vec{q}_{e}, e\right)$ in $W_{h}$. Define

$$
J_{h} e=E_{h} \Pi_{h}^{W} e
$$

for all $e$ in $R(E)$. Using these notations, we begin the analysis with the following two lemmas.

Lemma 4.2. $E_{h}$ is a self-adjoint operator in $L^{2}(\Omega)$.

Proof. By Lemma 2.2, $T_{h}$ is self-adjoint. It is well known that the spectral projection of any normal operator is self-adjoint [15. Since $E_{h}$ is the spectral projection of $T_{h}$, the lemma follows.

From now on, to simplify notation, let us abbreviate the norm $\|\cdot\|_{L^{2}(\Omega)}$ to simply $\|\cdot\|$, whenever it cannot be confused with the previously defined $L^{2}(\Omega)$-operator norm, which we continue to also denote by $\|\cdot\|$.

Lemma 4.3. Suppose (20) holds. Then, there exists an $h_{0}>0$ such that for all $h<h_{0}$, the operators $J_{h}: R(E) \longmapsto R\left(E_{h}\right)$ and $\left.E_{h}\right|_{R(E)}: R(E) \longmapsto R\left(E_{h}\right)$ are bijections, and there are $h$-independent constants $C_{\tau, j}$ such that

$$
\begin{aligned}
& C_{\tau, 1}\|e\| \leq\left\|J_{h} e\right\| \leq C_{\tau, 2}\|e\|, \\
& C_{\tau, 3}\|e\| \leq\left\|E_{h} e\right\| \leq\|e\|,
\end{aligned}
$$

for all e in $R(E)$.

Proof. By item 4 of Corollary 3.2, the gap between $R(E)$ and $R\left(E_{h}\right)$ becomes less than one when $h$ is small enough and, consequently, $\operatorname{dim}(R(E))=\operatorname{dim}\left(R\left(E_{h}\right)\right.$ ) (see, e.g., [14, Lemma 221]). Therefore, to prove the stated bijectivity, we only need to prove injectivity.

In preparation, we recall that by Lemma 4.2, $E_{h}$ is self-adjoint, and by Corollary [3.2(2), $E_{h}$ is a projector. Hence $E_{h}$ is an orthogonal projector. Orthogonal projectors have unit norm, hence

$$
\left\|E_{h} v\right\| \leq\|v\|, \quad \forall v \in L^{2}(\Omega) .
$$

A second preparatory inequality we need is

$$
\left\|e-\Pi_{h}^{W} e\right\| \leq C_{\tau} h^{r}\|e\|, \quad \forall e \in R(E),
$$

with $r=\min \left(s_{\mu}, k+1\right)$. This is a consequence of (11b), by which

$$
\left\|e-\Pi_{h}^{W} e\right\| \leq C_{\tau} h^{r}\left(|e|_{H^{s_{\mu}(\Omega)}}+\left|\overrightarrow{q_{e}}\right|_{H^{s^{\mu}}(\Omega)}\right),
$$

where $\vec{q}_{e}$ is as in (22). Thus, (26) follows from the regularity assumption (20). 
Let us now prove (23), beginning with the lower bound

$$
\begin{aligned}
\left\|J_{h} e\right\| & \geq\|e\|-\left\|e-E_{h} \Pi_{h}^{W} e\right\| & & \\
& =\|e\|-\left\|\left(E e-E_{h} e\right)+E_{h}\left(e-\Pi_{h}^{W} e\right)\right\| & & \text { since } e \in R(E) \\
& \geq\|e\|-\left\|\left(E-E_{h}\right) e\right\|-\left\|e-\Pi_{h}^{W} e\right\| & & \text { by (25) } \\
& \geq\left(1-C_{\tau} h^{r}\right)\|e\| & & \text { by (26) and (19). }
\end{aligned}
$$

Therefore, the lower bound follows by choosing small enough $h$. The injectivity of $J_{h}$ is an obvious consequence of this lower bound. The upper bound of (23) immediately follows by combining (26),

$$
\left\|\Pi_{h}^{W} e\right\| \leq\left(1+C_{\tau} h^{r}\right)\|e\| \quad \forall e \in R(E)
$$

with (25).

To finish the proof, note that the upper bound in (24) is already proved in (25). The lower bound in (24) (and the consequent injectivity of $E_{h}$ on $R(E)$ ) follows by an simpler argument similar to the above.

Proof of Theorem 4.1. As a first step, we define two finite dimensional operators whose eigenvalues are $\mu$ and $\mu_{h, j}$. Let $h$ be so small that we can conclude by Lemma4.3 that $J_{h}^{-1}: R\left(E_{h}\right) \rightarrow R(E)$ exists. Set $\hat{T}=\left.T\right|_{R(E)}$ and $\hat{T}_{h}=\left.J_{h}^{-1} T_{h} J_{h}\right|_{R(E)}$. Both the operators

$$
\hat{T}: R(E) \rightarrow R(E) \quad \text { and } \quad \hat{T}_{h}: R(E) \rightarrow R(E)
$$

are finite dimensional. $\hat{T}$ has $\mu$ as its (only) eigenvalue of of multiplicity $m$. Moreover, it is easy to see that $\mu_{h, j}$ 's are the eigenvalues of $\hat{T}_{h}$. Hence, by the Bauer-Fike theorem [3],

$$
\left|\mu-\mu_{h, j}\right| \leq C\left\|\hat{T}-\hat{T}_{h}\right\|
$$

The remainder of the proof bounds the above right-hand side appropriately.

To this end, let $f \in R(E)$, and consider $\left(\hat{T}-\hat{T}_{h}\right) f$. Then,

$$
\begin{aligned}
C_{\tau, 1}\left\|\left(\hat{T}-\hat{T}_{h}\right) f\right\| & \leq\left\|J_{h}\left(\hat{T}-\hat{T}_{h}\right) f\right\| & \text { by (23) of Lemma } 4.3 \\
& =\left\|E_{h} \Pi_{h}^{W} T f-T_{h} E_{h} \Pi_{h}^{W} f\right\| & \\
& =\left\|E_{h}\left(\Pi_{h}^{W} T f-T_{h} \Pi_{h}^{W} f\right)\right\| & \text { as } T_{h} \text { and } E_{h} \text { commute. }
\end{aligned}
$$

We bound the norm in (28) by duality, as follows. By Lemma 4.3, any $g_{h}$ in $R\left(E_{h}\right)$ can be written as $E_{h} g$ for some $g$ in $R(E)$. Therefore,

$$
\begin{aligned}
\left\|E_{h}\left(\Pi_{h}^{W} T f-T_{h} \Pi_{h}^{W} f\right)\right\| & =\sup _{g_{h} \in R\left(E_{h}\right)} \frac{\left(E_{h}\left(\Pi_{h}^{W} T f-T_{h} \Pi_{h}^{W} f\right), g_{h}\right)}{\left\|g_{h}\right\|} \\
& =\sup _{g \in R(E)} \frac{\left(E_{h}\left(\Pi_{h}^{W} T f-T_{h} \Pi_{h}^{W} f\right), E_{h} g\right)}{\left\|E_{h} g\right\|} \\
& \leq \frac{1}{C_{\tau, 3}} \sup _{g \in R(E)} \frac{\left(\Pi_{h}^{W} T f-T_{h} \Pi_{h}^{W} f, E_{h} g\right)}{\|g\|} .
\end{aligned}
$$

Note that to obtain the numerator above, we used the self-adjointness of $E_{h}$ given by Lemma 4.2. while to obtain the denominator, we used Lemma 4.3 . 
It will now be convenient to split the numerator in (29) into several terms. With $f$ and $g$ in $R(E)$, we write

$$
\begin{aligned}
\left(\Pi_{h}^{W} T f-T_{h} \Pi_{h}^{W} f, E_{h} g\right) & =\left(\Pi_{h}^{W} T f-T_{h} f, E_{h} g\right)+\left(T_{h}\left(f-\Pi_{h}^{W} f\right), E_{h} g\right) \\
& =t_{1}+t_{2}+t_{3}+t_{4}
\end{aligned}
$$

where

$$
\begin{aligned}
& t_{1}=\left(\Pi_{h}^{W} T f-T_{h} f, E_{h} g-g\right), \\
& t_{2}=\left(\Pi_{h}^{W} T f-T_{h} f, g\right), \\
& t_{3}=\left(T_{h}\left(f-\Pi_{h}^{W} f\right), E_{h} g-g\right), \\
& t_{4}=\left(T_{h}\left(f-\Pi_{h}^{W} f\right), g\right),
\end{aligned}
$$

and proceed to estimate the $t_{i}$ 's, beginning with $t_{1}$. Let $r_{1}=\min \left(s_{\mu}, k+1\right)$. We use Theorem 2.1] and (19) to get

$$
\begin{aligned}
t_{1} & =\left(\Pi_{h}^{W} u^{f}-u_{h}^{f},\left(E_{h}-E\right) g\right) \\
& \leq C_{\tau} h^{r_{1}}\left(\left|u^{f}\right|_{H^{r_{1}(\Omega)}}+\left|q^{f}\right|_{H^{r_{1}(\Omega)}}\right) h^{r_{1}}\|g\| \\
& \leq C_{\tau} h^{2 r_{1}}\|f\|\|g\|,
\end{aligned}
$$

by (20).

Next, for $t_{2}$, we use a previously known duality identity [10, Lemma 4.1] by which

$$
t_{2}=\left(\Pi_{h}^{W} u^{f}-u_{h}^{f}, g\right)=\left(c\left(\vec{q}^{f}-\vec{q}_{h}^{f}\right), \Pi_{h}^{V} \vec{q}^{g}-\vec{q}^{g}\right)+\left(\vec{q}^{f}-\Pi_{h}^{V} \vec{q}^{f}, \vec{\nabla} u^{g}-\vec{\nabla} w_{h}\right) \mathscr{T}_{h}
$$

for any $w_{h} \in W_{h}$. The first term on the right-hand side can be bounded by $C_{\tau} h^{2 r_{1}}\|f\|\|g\|$ using (11) and Theorem 2.1. For the second term, we use the standard result

$$
\inf _{w_{h} \in W_{h}}\left\|\vec{\nabla} u^{g}-\vec{\nabla} w_{h}\right\| \leq C h^{r_{0}}\left|u^{g}\right|_{H^{r_{0}+1}(\Omega)}
$$

with $r_{0}=\min \left(s_{\mu}, k\right)$, to get

$$
\begin{aligned}
t_{2} & \leq C_{\tau} h^{2 r_{1}}\|f\|\|g\|+C_{\tau} h^{r_{1}}\left(\left|u^{f}\right|_{H^{r_{1}}(\Omega)}+\left|q^{f}\right|_{H^{r_{1}}(\Omega)}\right) h^{r_{0}}\left|u^{g}\right|_{H^{r_{0}+1}(\Omega)} \\
& \leq C_{\tau}\left(h^{2 r_{1}}+h^{r_{1}+r_{0}}\right)\|f\|\|g\|
\end{aligned}
$$

by the regularity assumption (20).

Now consider $t_{3}$. Since $T$ is a bounded operator in $L^{2}(\Omega)$, by Theorem 3.1, we have

$$
\left\|T_{h} v\right\| \leq C_{\tau}\|v\| \quad \forall v \in L^{2}(\Omega) .
$$

Hence,

$$
t_{3}=\left(T_{h}\left(f-\Pi_{h}^{W} f\right),\left(E_{h}-E\right) g\right) \leq C_{\tau}\left\|f-\Pi_{h}^{W} f\right\| C h^{r_{1}}\|g\|
$$

by (19) and (20). To control $f-\Pi_{h}^{W} f$ let us first note that $u^{f}=\mu f$ since $f \in R(E)$. Hence absorbing the $\mu$-dependence into $C_{\tau}$, we can write

$$
\left\|f-\Pi_{h}^{W} f\right\| \leq C_{\tau} h^{r_{1}}\left(\left|u^{f}\right|_{H^{r_{1}(\Omega)}}+\left|q^{f}\right|_{H^{r_{1}(\Omega)}}\right) .
$$

Using the regularity assumption (20) again, we conclude that

$$
t_{3} \leq C h^{2 r_{1}}\|f\|\|g\| \text {. }
$$


Finally, consider $t_{4}$. By Lemma 2.2. $T_{h}$ is self-adjoint, so

$$
\begin{aligned}
t_{4} & =\left(f-\Pi_{h}^{W} f, u_{h}^{g}\right) \\
& =\left(f-\Pi_{h}^{W} f, u_{h}^{g}-u_{h, k-1}^{g}\right)
\end{aligned}
$$

where $u_{k-1}^{g} \in L^{2}(\Omega)$ is the solution of the HDG method using polynomials of degree $k-1$ in place of $k$ for all $k \geq 1$, while when $k=0$, we set $u_{k-1}^{g}=0$. We may introduce $u_{k-1}^{g}$ in (32) because of (10b). By Theorem 2.1 and (20),

$$
\left\|u_{h}^{g}-u_{h, k-1}^{g}\right\| \leq\left\|u_{h}^{g}-u\right\|+\left\|u-u_{h, k-1}^{g}\right\| \leq C_{\tau} h^{r_{0}}\|g\| .
$$

Hence, using (31) also, we find that

$$
t_{4} \leq C_{\tau} h^{r_{0}+r_{1}}\|f\|\|g\| .
$$

Combining the estimates for all $t_{i}$ to bound the right-hand side of (29), we find from (28) that

$$
\left\|\left(\hat{T}-\hat{T}_{h}\right) f\right\| \leq C_{\tau} h^{r_{0}+r_{1}}\|f\| .
$$

Using this in (27) the proof is finished.

\section{THE HYBRIDIZED EIGENPROBLEM}

The main advantage the HDG method possesses over other DG methods, in source problems, is that one can eliminate (all) the interior variables $\left(u_{h}^{f}, \vec{q}_{h}^{f}\right)$ to obtain a single equation for the Lagrange multiplier $\eta_{h}^{f}$. Since $\eta_{h}^{f}$ is defined on mesh faces of dimension $n-1$, the reduced system for $\eta_{h}^{f}$ can be significantly smaller in size for high degrees $k$. It is natural to ask if this reduction in system size can be carried over to the eigenproblem. In this section we study this issue. The analysis here is modeled after 9 .

First, let us review the above mentioned elimination result for the source problem. Define local solution operators $\overrightarrow{\mathcal{Q}}: M_{h} \rightarrow V_{h}, \mathcal{U}: M_{h} \rightarrow W_{h}, \vec{Q}_{W}: L^{2}(\Omega) \rightarrow V_{h}$, $\mathcal{U}_{W}: L^{2}(\Omega) \rightarrow W_{h}$, element by element, by the following two systems: For any $K$ in $\mathscr{T}_{h}$,

$$
\begin{aligned}
(c \vec{Q} \mu, \vec{r})_{K}-(\mathcal{U} \mu, \nabla \cdot \vec{r})_{K} & =-\langle\mu, \vec{r} \cdot \vec{n}\rangle_{\partial K} & & \text { for all } \vec{r} \in V_{h}, \\
(w, \nabla \cdot \vec{Q} \mu)_{K}+\langle\tau(\mathcal{U} \mu-\mu), w\rangle_{\partial K} & =0 & & \text { for all } w \in W_{h}
\end{aligned}
$$

and

$$
\begin{aligned}
\left(c \vec{Q}_{W} f, \vec{r}\right)_{K}-\left(\mathcal{U}_{W} f, \nabla \cdot \vec{r}\right)_{K} & =0 & & \text { for all } \vec{r} \in V_{h}, \\
\left(w, \nabla \cdot \vec{Q}_{W} f\right)_{K}+\left\langle\tau \mathcal{U}_{W} f, w\right\rangle_{\partial K} & =(f, w)_{K} & & \text { for all } w \in W_{h} .
\end{aligned}
$$

The properties of these operators are amply discussed in [7, 8, and will not be repeated. Let $a_{h}(\eta, \mu)=(c \vec{Q} \eta, \vec{Q} \mu)_{\mathscr{T}_{h}}+\langle\tau(\mathcal{U} \eta-\eta),(\mathcal{U} \mu-\mu)\rangle_{\partial \mathscr{T}_{h}}$ and $b_{h}(\mu)=$ $(f, \mathcal{U} \mu)_{\mathscr{T}_{h}}$.

Theorem 5.1 (The reduced source problem; see [8, Theorem 2.1]). The functions $\vec{q}_{h}^{f} \in V_{h}, u_{h}^{f} \in W_{h}$, and $\eta_{h}^{f} \in M_{h}$ satisfy (7) if and only if $\eta_{h}^{f}$ is the unique function in $M_{h}$ satisfying

$$
\begin{gathered}
a_{h}\left(\eta_{h}^{f}, \mu\right)=b_{h}(\mu) \quad \text { for all } \mu \in M_{h}, \\
\vec{q}_{h}^{f}=\vec{Q} \eta_{h}^{f}+\vec{Q}_{W} f \quad \text { and } \quad u_{h}^{f}=\mathcal{U} \eta_{h}^{f}+\mathcal{U}_{W} f .
\end{gathered}
$$


Our aim is to obtain an analogue of Theorem 5.1 for the eigenproblem. To this end, we will need the next lemma. Let

$$
d_{h \tau}^{K}=1+\left(\tau_{K}^{\max } h_{K}\right)^{-1 / 2}, \quad c_{h \tau}^{K}=1+\left(\tau_{K}^{*} h_{K}\right)^{1 / 2}, \quad\|\mu\|_{h, K}^{2}=\|\mu\|_{L^{2}(\partial K)}^{2} \frac{|K|}{|\partial K|} .
$$

We assume throughout this section there is a mesh-independent $C_{*}>0$ such that

$$
\left(d_{h \tau}^{K}\right)^{2} h_{K} \leq C_{*} \quad \text { and } \quad c_{h \tau}^{K} \leq C_{*} .
$$

This certainly holds for the most commonly used choice of $\tau \equiv 1$.

Lemma 5.2. The local solution operators satisfy the following bounds: There are positive constants $C_{0}$ and $C_{1}$ (independent of $h_{K}$ ) such that

$$
\begin{array}{rlrl}
\left\|\mathcal{U}_{W} f\right\|_{L^{2}(K)} & \leq C_{0}\left(d_{h \tau}^{K}\right)^{2} h_{K}^{2}\|f\|_{L^{2}(K)} & & \forall f \in L^{2}(K), \\
\left\|\mathcal{U}_{\mu}\right\|_{L^{2}(K)} \leq C_{1} c_{h \tau}^{K}\|\mu\|_{h, K} & & \forall \mu \in M_{h} .
\end{array}
$$

Moreover, whenever $0<\kappa<1 /\left(C_{0}\left(d_{h \tau}^{K}\right)^{2} h_{K}^{2}\right)$, the operator $I-\kappa \mathcal{U}_{W}$ is invertible and

$$
\left\|\left(I-\kappa \mathcal{U}_{W}\right)^{-1} f\right\|_{L^{2}(K)} \leq \frac{1}{1-\kappa C_{0}\left(d_{h \tau}^{K}\right)^{2} h_{K}^{2}}\|f\|_{L^{2}(K)} \quad \forall f \in L^{2}(K) .
$$

Proof. The estimates (37) and (38) are proved in [7]. To prove (39), we use (37), by which

$$
\left\|\kappa \mathcal{U}_{W} f\right\|_{L^{2}(K)} \leq \gamma\|f\|_{L^{2}(K)}
$$

where $\gamma=\kappa\left(d_{h \tau}^{K}\right)^{2} C_{0} h_{K}^{2}$. By the given assumption $\gamma<1$, so the $L^{2}(\Omega)$-operator norm of $\kappa \mathcal{U}_{W}$ is less than one. Consequently, $I-\kappa \mathcal{U}_{W}$ is invertible and the norm of the inverse is less than $(1-\gamma)^{-1}$.

Theorem 5.3. Let $0<C_{3}<\left(C_{0} C_{*}\right)^{-1}$ and let $\lambda_{h}$ be any positive number less than $C_{3} / h$. Then $I-\lambda_{h} \mathcal{U}_{W}$ is invertible, and moreover, $\lambda_{h}$ satisfies

$$
a_{h}\left(\eta_{h}, \mu\right)=\lambda_{h}\left(\left(I-\lambda_{h} \mathcal{U}_{W}\right)^{-1} \mathcal{U}_{h}, \mathcal{U} \mu\right)_{\mathscr{T}_{h}} \quad \forall \mu \in M_{h}
$$

with some nontrivial $\eta_{h}$ in $M_{h}$, if and only if the number $\lambda_{h}$ and the functions

$$
\eta_{h}, \quad u_{h}=\left(I-\lambda_{h} \mathcal{U}_{W}\right)^{-1} \mathcal{U} \eta_{h}, \quad \text { and } \quad q_{h}=\overrightarrow{\mathcal{Q}} \eta_{h}+\lambda_{h} \vec{Q}_{W} u_{h}
$$

together solve the HDG eigenproblem (14).

Proof. The argument proceeds as in [9, so we will be brief. Setting $f=\lambda_{h} u_{h}$ in (7) and applying Theorem 5.1, (36), we get $u_{h}=\mathcal{U}_{\eta_{h}}+\mathcal{U}_{W}\left(\lambda_{h} u_{h}\right)$. Hence,

$$
u_{h}=\left(I-\lambda_{h} \mathcal{u}_{W}\right)^{-1} \mathcal{U}_{h},
$$

where the inverse exists by Lemma [5.2, whenever $\lambda_{h}<1 /\left(C_{0}\left(d_{h \tau}^{K}\right)^{2} h_{K}^{2}\right)$. Note that the later inequality is satisfied whenever $\lambda_{h}<C_{3} / h$ because $\lambda_{h} C_{0} d_{h \tau}^{K} h_{K}^{2} \leq$ $\lambda_{h} C_{0} C_{*} h_{K} \leq \lambda_{h} C_{0} C_{*} h<C_{3} C_{0} C_{*}<1$. Now, using (42) in the right-hand side of (35) and proceeding as in [9, the proof is finished.

Theorem 5.3 is the analogue of Theorem 5.1 for the eigenproblem. It shows, roughly speaking, that the reduced form of the eigenproblem, namely (40), does not lose eigenvalues up to $O(1 / h)$. In particular, the physically important lower range of the spectrum is preserved. The difficulty with (40) is that in spite of being a smaller system (14), it is a nonlinear eigenvalue problem. Fortunately, the result we present next shows that good initial guesses for the nonlinear eigenproblem (40) 
can be calculated by solving a standard symmetric generalized eigenproblem. This standard eigenproblem is to find an eigenvalue $\tilde{\lambda}_{h}>0$ and a corresponding eigenfunction $\tilde{\eta}_{h} \not \equiv 0$ in $M_{h}$ satisfying

$$
a_{h}\left(\tilde{\eta}_{h}, \mu\right)=\tilde{\lambda}_{h}\left(\mathcal{U} \tilde{\eta}_{h}, \mathcal{U} \mu\right)_{\mathscr{T}_{h}} \quad \forall \mu \in M_{h}
$$

and algorithms for solving (43) are well developed. Although $\tilde{\lambda}_{h}$ may not equal $\lambda_{h}$, it is a good approximation to $\lambda_{h}$ (see Theorem 5.4) and hence can be used as an initial iterate for iterative algorithms (such as those studied in [9]) for the nonlinear eigenproblem.

Theorem 5.4. Let $0<C_{3}<\left(C_{0} C_{*}\right)^{-1}$ and consider any eigenvalue $\lambda_{h}$ of (14) satisfying $\lambda_{h}<C_{3} / h$. Then there are two constants, $h_{0}>0$ (depending on $\lambda_{h}$ ) and $C>0$ (independent of $\lambda_{h}$ ), such that for all $h \leq h_{0}$, there is an eigenvalue $\tilde{\lambda}_{h}$ of (43) satisfying

$$
\frac{\left|\lambda_{h}-\tilde{\lambda}_{h}\right|}{\lambda_{h}} \leq C \lambda_{h} \tilde{\lambda}_{h} h .
$$

Proof. The idea, as in 9], is to compare two operators which have $\lambda_{h}$ and $\tilde{\lambda}_{h}$ as eigenvalues. The operators are $S_{h}^{(\kappa)}: M_{h} \rightarrow M_{h}$ and $\tilde{S}_{h}: M_{h} \rightarrow M_{h}$ defined by

$$
\begin{aligned}
a_{h}\left(S_{h}^{(\kappa)} \mu, \gamma\right) & =\left(\left(I-\kappa \mathcal{U}_{W}\right)^{-1} \mathcal{U} \mu, \mathcal{U} \gamma\right)_{\mathscr{T}_{h}} & & \forall \gamma \in M_{h}, \\
a_{h}\left(\tilde{S}_{h} \mu, \gamma\right) & =(\mathcal{U} \mu, \mathcal{U} \gamma)_{\mathscr{T}_{h}} & & \forall \gamma \in M_{h} .
\end{aligned}
$$

We will use $S_{h}^{(\kappa)}$ with $\kappa=\lambda_{h}$, noting that the inverse of $I-\lambda_{h} \mathcal{U}_{W}$ appearing in the definition of $S_{h}^{\left(\lambda_{h}\right)}$ exists since $\lambda_{h}<C_{3} / h$ (due to Lemma 5.2. see also proof of Theorem [5.3). Both operators are self-adjoint in the $a_{h}(\cdot, \cdot)$-inner product: The self-adjointness of $\tilde{S}_{h}$ is obvious. It is also easy to see that $\left(f, \mathcal{U}_{W} g\right)_{K}=$ $\left(c \vec{Q}_{W} g, \vec{Q}_{W} f\right)_{K}+\left\langle\tau \mathcal{U}_{W} f, \mathcal{U}_{W} g\right\rangle_{\partial K}$ due to (33), so $\mathcal{U}_{W}$ in self-adjoint in the $L^{2}(\Omega)$ inner product. Therefore, $S_{h}^{\left(\lambda_{h}\right)}$ is self-adjoint in the $a_{h}(\cdot, \cdot)$-inner product.

Now, (40) and (43) imply, respectively, that

$$
\lambda_{h}^{-1} \in \sigma\left(S_{h}^{\left(\lambda_{h}\right)}\right) \quad \text { and } \quad \tilde{\lambda}_{h}^{-1} \in \sigma\left(\tilde{S}_{h}\right),
$$

hence, by Weyl's theorem [19] on eigenvalues of self-adjoint operators, we conclude that

$$
\left|\lambda_{h}^{-1}-\tilde{\lambda}_{h}^{-1}\right| \leq\left\|S_{h}^{\left(\lambda_{h}\right)}-\tilde{S}_{h}\right\|_{a} \equiv \sup _{0 \neq \gamma, \mu \in M_{h}} \frac{a_{h}\left(\left(S_{h}^{\left(\lambda_{h}\right)}-\tilde{S}_{h}\right) \gamma, \mu\right)}{a_{h}(\gamma, \gamma)^{1 / 2} a_{h}(\mu, \mu)^{1 / 2}} .
$$

In the remainder of the proof, we bound the right-hand side.

By Lemma 5.2 choosing $h_{0}$ appropriately small, we have, for all $h \leq h_{0}$,

$$
\begin{aligned}
a_{h}\left(\left(S_{h}^{\left(\lambda_{h}\right)}-\tilde{S}_{h}\right) \gamma, \mu\right) & =\left(\lambda_{h} \mathcal{U}_{W}\left(I-\lambda_{h} \mathcal{U}_{W}\right)^{-1} \mathcal{U} \gamma, \mathcal{U} \mu\right) & & \\
& \leq \frac{\lambda_{h} C_{0} C_{*} h_{K}}{1-\lambda_{h} C_{0} C_{*} h_{K}}\|\mathcal{U} \gamma\|\|\mathcal{U} \mu\| & & \text { by (37) and (39), } \\
& \leq C \lambda_{h} h\|\mathcal{U} \gamma\|\left\|\mathcal{U}_{\mu}\right\| & & \\
& \leq C \lambda_{h} h\|\gamma\|_{h}\|\mu\|_{h} & & \text { by (38), }
\end{aligned}
$$

where $\|\mu\|_{h}^{2}=\sum_{K}\|\mu\|_{h, K}^{2}$. We now use the Poincaré-type estimate [7, Theorem 3.4], $\|\mu\|_{h}^{2} \leq C a_{h}(\mu, \mu)$ to conclude that

$$
a_{h}\left(\left(S_{h}^{\left(\lambda_{h}\right)}-\tilde{S}_{h}\right) \gamma, \mu\right) \leq C \lambda_{h} h a_{h}(\gamma, \gamma)^{1 / 2} a_{h}(\mu, \mu)^{1 / 2} .
$$

Returning to (45) and using this estimate, the theorem is proved. 


\section{NUMERICAL EXPERIMENTS}

In this section, we present numerical results to illustrate the theoretical results of the previous sections. We consider model eigenproblems on a square and an L-shaped domain and compute the spectral approximations using the HDG discretization. The model problems are the same as those considered in [9] so as to facilitate comparison with the HRT mixed method. We also present a local postprocessing technique that enhances the eigenfunction and eigenvalue accuracy beyond the convergence orders predicted by the theory. We begin by describing this postprocessing in the next subsection and present the numerical results in the later subsections.

6.1. Local postprocessing. To postprocess the eigenfunction, we are motivated by the theoretical results that show that the approximate gradient $\vec{q}_{h}$ converges at the same order as the approximate eigenfunction $u_{h}$. Accordingly, following [18, we define (element by element) the postprocessed eigenfunction $u_{h}^{*} \in P_{k+1}(K)$ by

$$
\begin{aligned}
\left(\vec{\nabla} u_{h}^{*}, \vec{\nabla} w\right)_{K} & =-\left(c \vec{q}_{h}, \vec{\nabla} w\right)_{K}, \quad \forall w \in P_{k+1}(K), \\
\left(u_{h}^{*}, 1\right)_{K} & =\left(u_{h}, 1\right)_{K},
\end{aligned}
$$

for all elements $K \in \mathscr{T}_{h}$. A convergence theory for this postprocessing (that predicts that $u_{h}^{*}$ converges at the rate $O\left(h^{k+2}\right)$ for $k \geq 1$ ) is available for solutions of the source problem [18] and for the HRT mixed eigenproblem [9]. Next, we define a postprocessed eigenflux $\vec{q}_{h}^{*}$ as the unique element of $\left[P_{k}(K)\right]^{n}+\vec{x} P_{k}(K)$ satisfying

$$
\begin{aligned}
\left\langle\left(\vec{q}_{h}^{*}-\widehat{q}_{h}\right) \cdot \vec{n}, \mu\right\rangle_{F} & =0, \quad \forall \mu \in P_{k}(F), \forall F \subseteq \partial K, \\
\left(\vec{q}_{h}^{*}-\vec{q}_{h}, \vec{v}\right)_{K} & =0, \quad \forall \vec{v} \in\left[P_{k-1}(K)\right]^{d} \quad \text { if } k \geq 1,
\end{aligned}
$$

for all elements $K \in \mathscr{T}_{h}$. Note that $\vec{q}_{h}^{*}$ is $H(\operatorname{div}, \Omega)$-conforming.

Using this postprocessed eigenfunction and eigenflux, we are now motivated by the Rayleigh quotient to define the following expression for computing an approximate eigenvalue:

$$
\lambda_{h}^{*}=\frac{\left(\alpha \vec{\nabla} u_{h}^{*}, \vec{\nabla} u_{h}^{*}\right)_{\mathscr{T}_{h}}+\left\langle\vec{q}_{h}^{*} \cdot \vec{n}, u_{h}^{*}\right\rangle_{\partial \mathscr{T}_{h}}}{\left(u_{h}^{*}, u_{h}^{*}\right)_{\mathscr{T}_{h}}} .
$$

As the numerical results below indicate, this postprocessed eigenvalue $\lambda_{h}^{*}$ can be a superior approximation to $\lambda_{h}$.

6.2. Square domain. We consider the domain $\Omega=(0, \pi) \times(0, \pi)$. In this case, the exact eigenvalues and eigenfunctions are given by $\lambda^{m n}=m^{2}+n^{2}$ and $u^{m n}=$ $\sin (m x) \sin (n x)$, respectively, for $m, n \in \mathbb{N}_{+}$. Clearly, the eigenfunctions are infinitely smooth, so the convergence rates should be limited only by the degrees of the approximating polynomials. We obtain an initial mesh by subdividing $\Omega$ into a uniform grid of $4 \times 4$ squares and splitting each square into two triangles by its positively sloped diagonal. Successively finer meshes are obtained by subdividing each triangle into four smaller triangles. The mesh at "level $\ell$ " is obtained from the initial mesh by $\ell$ refinements. We compute the solution of the HDG eigenproblem in each of these meshes. The results obtained are collected below. 
TABLE 1. Convergence of the approximate eigenvalues $\lambda_{h}$ for $\tau=1$.

\begin{tabular}{|c|c||cc|cc|cc|cc|}
\hline $\begin{array}{c}\text { degree } \\
k\end{array}$ & mesh & \multicolumn{2}{c|}{ first mode } & \multicolumn{2}{c|}{ second mode } & \multicolumn{2}{c|}{ fourth mode } & \multicolumn{2}{c|}{ sixth mode } \\
error & $\ell$ & order & error & order & error & order & error & order \\
\hline \multirow{4}{*}{0} & 0 & $4.49 \mathrm{e}-2$ & -- & $1.26 \mathrm{e}-0$ & -- & $3.03 \mathrm{e}-0$ & -- & $4.58 \mathrm{e}-0$ & -- \\
& 1 & $3.45 \mathrm{e}-2$ & 0.38 & $7.11 \mathrm{e}-1$ & 0.82 & $1.86 \mathrm{e}-0$ & 0.70 & $3.00 \mathrm{e}-0$ & 0.61 \\
& 2 & $2.05 \mathrm{e}-2$ & 0.75 & $3.82 \mathrm{e}-1$ & 0.90 & $1.07 \mathrm{e}-0$ & 0.79 & $1.78 \mathrm{e}-0$ & 0.75 \\
& 3 & $1.11 \mathrm{e}-2$ & 0.89 & $1.99 \mathrm{e}-1$ & 0.94 & $5.84 \mathrm{e}-1$ & 0.88 & $9.81 \mathrm{e}-1$ & 0.86 \\
& 4 & $5.74 \mathrm{e}-3$ & 0.95 & $1.01 \mathrm{e}-1$ & 0.97 & $3.06 \mathrm{e}-1$ & 0.93 & $5.16 \mathrm{e}-1$ & 0.93 \\
\hline \multirow{4}{*}{1} & 0 & $5.97 \mathrm{e}-3$ & -- & $1.40 \mathrm{e}-1$ & -- & $7.35 \mathrm{e}-1$ & -- & $1.77 \mathrm{e}-0$ & -- \\
& 1 & $8.44 \mathrm{e}-4$ & 2.82 & $1.58 \mathrm{e}-2$ & 3.14 & $9.52 \mathrm{e}-2$ & 2.95 & $2.36 \mathrm{e}-1$ & 2.91 \\
& 2 & $1.10 \mathrm{e}-4$ & 2.94 & $1.84 \mathrm{e}-3$ & 3.11 & $1.11 \mathrm{e}-2$ & 3.10 & $2.65 \mathrm{e}-2$ & 3.15 \\
& 3 & $1.39 \mathrm{e}-5$ & 2.98 & $2.20 \mathrm{e}-4$ & 3.06 & $1.32 \mathrm{e}-3$ & 3.07 & $3.08 \mathrm{e}-3$ & 3.10 \\
& 4 & $1.75 \mathrm{e}-6$ & 2.99 & $2.69 \mathrm{e}-5$ & 3.03 & $1.61 \mathrm{e}-4$ & 3.04 & $3.71 \mathrm{e}-4$ & 3.06 \\
\hline \multirow{4}{*}{2} & 0 & $1.38 \mathrm{e}-4$ & -- & $3.65 \mathrm{e}-3$ & -- & $4.15 \mathrm{e}-2$ & -- & $1.27 \mathrm{e}-1$ & -- \\
& 1 & $4.53 \mathrm{e}-6$ & 4.93 & $1.04 \mathrm{e}-4$ & 5.13 & $1.26 \mathrm{e}-3$ & 5.04 & $3.51 \mathrm{e}-3$ & 5.18 \\
& 2 & $1.43 \mathrm{e}-7$ & 4.98 & $3.12 \mathrm{e}-6$ & 5.06 & $3.82 \mathrm{e}-5$ & 5.05 & $1.02 \mathrm{e}-4$ & 5.10 \\
& 3 & $4.50 \mathrm{e}-9$ & 4.99 & $9.52 \mathrm{e}-8$ & 5.03 & $1.17 \mathrm{e}-6$ & 5.03 & $3.06 \mathrm{e}-6$ & 5.06 \\
& 4 & $1.41 \mathrm{e}-10$ & 5.00 & $2.94 \mathrm{e}-9$ & 5.02 & $3.60 \mathrm{e}-8$ & 5.02 & $9.37 \mathrm{e}-8$ & 5.03 \\
\hline
\end{tabular}

TABle 2. Convergence of the postprocessed eigenvalues $\lambda_{h}^{*}$ for $\tau=1$.

\begin{tabular}{|c|c||cc|cc|cc|cc|}
\hline $\begin{array}{c}\text { degree } \\
k\end{array}$ & mesh & \multicolumn{2}{c|}{ first mode } & \multicolumn{2}{c|}{ second mode } & \multicolumn{2}{c|}{ fourth mode } & \multicolumn{2}{c|}{ sixth mode } \\
error & order & error & order & error & order & error & order \\
\hline \multirow{4}{*}{0} & 0 & $3.42 \mathrm{e}-1$ & -- & $1.79 \mathrm{e}-0$ & -- & $3.56 \mathrm{e}-0$ & -- & $5.23 \mathrm{e}-0$ & -- \\
& 1 & $2.04 \mathrm{e}-1$ & 0.75 & $1.13 \mathrm{e}-0$ & 0.66 & $2.42 \mathrm{e}-0$ & 0.55 & $3.69 \mathrm{e}-0$ & 0.50 \\
& 2 & $1.09 \mathrm{e}-1$ & 0.90 & $6.40 \mathrm{e}-1$ & 0.82 & $1.48 \mathrm{e}-0$ & 0.71 & $2.28 \mathrm{e}-0$ & 0.70 \\
& 3 & $5.62 \mathrm{e}-2$ & 0.96 & $3.40 \mathrm{e}-1$ & 0.91 & $8.25 \mathrm{e}-1$ & 0.84 & $1.28 \mathrm{e}-0$ & 0.83 \\
& 4 & $2.84 \mathrm{e}-2$ & 0.98 & $1.75 \mathrm{e}-1$ & 0.96 & $4.36 \mathrm{e}-1$ & 0.92 & $6.78 \mathrm{e}-1$ & 0.92 \\
\hline \multirow{4}{*}{1} & 0 & $2.64 \mathrm{e}-3$ & -- & $2.75 \mathrm{e}-2$ & -- & $1.93 \mathrm{e}-1$ & -- & $5.04 \mathrm{e}-1$ & -- \\
& 1 & $1.51 \mathrm{e}-4$ & 4.12 & $1.03 \mathrm{e}-3$ & 4.74 & $6.60 \mathrm{e}-3$ & 4.87 & $1.20 \mathrm{e}-2$ & 5.39 \\
& 2 & $8.95 \mathrm{e}-6$ & 4.08 & $4.88 \mathrm{e}-5$ & 4.39 & $2.56 \mathrm{e}-4$ & 4.69 & $3.79 \mathrm{e}-4$ & 4.98 \\
& 3 & $5.42 \mathrm{e}-7$ & 4.05 & $2.65 \mathrm{e}-6$ & 4.20 & $1.18 \mathrm{e}-5$ & 4.44 & $1.57 \mathrm{e}-5$ & 4.60 \\
& 4 & $3.33 \mathrm{e}-8$ & 4.02 & $1.54 \mathrm{e}-7$ & 4.10 & $6.17 \mathrm{e}-7$ & 4.25 & $7.73 \mathrm{e}-7$ & 4.34 \\
\hline \multirow{4}{*}{2} & 0 & $5.40 \mathrm{e}-5$ & -- & $7.03 \mathrm{e}-4$ & -- & $1.06 \mathrm{e}-2$ & -- & $2.14 \mathrm{e}-2$ & -- \\
& 1 & $8.04 \mathrm{e}-7$ & 6.07 & $9.39 \mathrm{e}-6$ & 6.23 & $1.43 \mathrm{e}-4$ & 6.20 & $2.14 \mathrm{e}-4$ & 6.64 \\
& 2 & $1.11 \mathrm{e}-8$ & 6.18 & $1.09 \mathrm{e}-7$ & 6.43 & $2.01 \mathrm{e}-6$ & 6.16 & $2.73 \mathrm{e}-6$ & 6.29 \\
& 3 & $1.28 \mathrm{e}-10$ & 6.44 & $1.32 \mathrm{e}-9$ & 6.37 & $2.74 \mathrm{e}-8$ & 6.20 & $3.34 \mathrm{e}-8$ & 6.35 \\
& 4 & $1.56 \mathrm{e}-12$ & 6.35 & $2.19 \mathrm{e}-11$ & 5.91 & $3.3 \mathrm{e}-10$ & 6.38 & $2.88 \mathrm{e}-10$ & 6.86 \\
\hline
\end{tabular}

In Table 1, the error and order of convergence of the approximate eigenvalues for $\tau=1$ is presented. We see that the approximate eigenvalues $\lambda_{h}$ converge to the exact values at the rate of $O\left(h^{2 k+1}\right)$. This is in good agreement with the theoretical result of Theorem 4.1. To compare with the HRT result, see [9, Table 1], which shows that the HRT eigenvalues converge faster at the rate $O\left(h^{2 k+2}\right)$.

Curiously, however, we found that the simple local postprocessing (48) can give eigenvalues competitive with the HRT eigenvalues. In Table 2, we present the error and order of convergence of the postprocessed eigenvalues $\lambda_{h}^{*}$. There is no change in the convergence order for $k=0$ as both the approximate and postprocessed eigenvalues converge linearly. However, observe that when $k \geq 1$, the postprocessed eigenvalues converge at a faster rate of $O\left(h^{2 k+2}\right)$. Presently, we do not have a rigorous proof for this convergence rate. This seems to be the fastest rate we can expect. Indeed, we also observed, in unreported experiments, that when the postprocessing (48) is applied to the HRT method, no further improvement in the 
TABLE 3. Convergence of the approximate $\left(u_{h}\right)$ and postprocessed $\left(u_{h}^{*}\right)$ eigenfunctions for $\tau=1$.

\begin{tabular}{|c|c|c|c|c|c|c|c|c|c|}
\hline \multicolumn{2}{|c|}{ eigenmode } & \multicolumn{4}{|c|}{ first } & \multicolumn{4}{|c|}{ fourth } \\
\hline $\begin{array}{c}\text { degree } \\
k\end{array}$ & $\begin{array}{c}\text { mesh } \\
\ell\end{array}$ & $\begin{array}{l}\| u-v \\
\text { error }\end{array}$ & $\begin{array}{l}\| \mathscr{T}_{h} \\
\text { order }\end{array}$ & $\begin{array}{l}\| u-u \\
\text { error }\end{array}$ & $\begin{array}{l}\| \mathscr{T}_{h} \\
\text { order }\end{array}$ & $\begin{array}{l}\| u-\imath \\
\text { error }\end{array}$ & $\begin{array}{l}\| \mathscr{T}_{h} \\
\text { order }\end{array}$ & $\begin{array}{l}\| u-\imath \\
\text { error }\end{array}$ & $\begin{array}{l}\| \mathscr{T}_{h} \\
\text { order }\end{array}$ \\
\hline \multirow{5}{*}{0} & 0 & $4.19 \mathrm{e}-2$ & -- & $8.65 \mathrm{e}-2$ & -- & $5.55 \mathrm{e}-1$ & -- & $6.11 \mathrm{e}-1$ & -- \\
\hline & 1 & $1.97 \mathrm{e}-2$ & 1.09 & $2.72 \mathrm{e}-2$ & 1.67 & $1.68 \mathrm{e}-1$ & 1.73 & $1.91 \mathrm{e}-1$ & 1.68 \\
\hline & 2 & $9.46 \mathrm{e}-3$ & 1.06 & $1.03 \mathrm{e}-2$ & 1.40 & $6.83 \mathrm{e}-2$ & 1.30 & $7.27 \mathrm{e}-2$ & 1.40 \\
\hline & 3 & $4.62 \mathrm{e}-3$ & 1.03 & $4.65 \mathrm{e}-3$ & 1.15 & $3.09 \mathrm{e}-2$ & 1.14 & $3.14 \mathrm{e}-2$ & 1.21 \\
\hline & 4 & $2.28 \mathrm{e}-3$ & 1.02 & $2.27 \mathrm{e}-3$ & 1.04 & $1.47 \mathrm{e}-2$ & 1.07 & $1.47 \mathrm{e}-2$ & 1.09 \\
\hline \multirow{5}{*}{1} & 0 & $5.02 \mathrm{e}-2$ & -- & $8.84 \mathrm{e}-3$ & -- & $3.21 \mathrm{e}-1$ & -- & $7.84 \mathrm{e}-2$ & -- \\
\hline & 1 & $1.26 \mathrm{e}-2$ & 2.00 & $1.14 \mathrm{e}-3$ & 2.96 & $8.10 \mathrm{e}-2$ & 1.99 & $9.23 \mathrm{e}-3$ & 3.09 \\
\hline & 2 & $3.14 \mathrm{e}-3$ & 2.00 & $1.44 \mathrm{e}-4$ & 2.98 & $1.92 \mathrm{e}-2$ & 2.07 & $1.13 \mathrm{e}-3$ & 3.03 \\
\hline & 3 & $7.85 \mathrm{e}-4$ & 2.00 & $1.82 \mathrm{e}-5$ & 2.99 & $4.67 \mathrm{e}-3$ & 2.04 & $1.39 \mathrm{e}-4$ & 3.02 \\
\hline & 4 & $1.96 \mathrm{e}-4$ & 2.00 & $2.28 \mathrm{e}-6$ & 2.99 & $1.15 \mathrm{e}-3$ & 2.02 & $1.72 \mathrm{e}-5$ & 3.01 \\
\hline \multirow{5}{*}{2} & 0 & $5.28 \mathrm{e}-3$ & -- & $8.36 \mathrm{e}-4$ & -- & $6.98 \mathrm{e}-2$ & -- & $1.31 \mathrm{e}-2$ & -- \\
\hline & 1 & $6.70 \mathrm{e}-4$ & 2.98 & $5.29 \mathrm{e}-5$ & 3.98 & $8.10 \mathrm{e}-3$ & 3.11 & $7.26 \mathrm{e}-4$ & 4.17 \\
\hline & 2 & $8.41 \mathrm{e}-5$ & 2.99 & $3.32 \mathrm{e}-6$ & 4.00 & $9.78 \mathrm{e}-4$ & 3.05 & $4.37 \mathrm{e}-5$ & 4.06 \\
\hline & 3 & $1.05 \mathrm{e}-5$ & 3.00 & $2.07 \mathrm{e}-7$ & 4.00 & $1.20 \mathrm{e}-4$ & 3.02 & $2.70 \mathrm{e}-6$ & 4.02 \\
\hline & 4 & $1.32 \mathrm{e}-6$ & 3.00 & $1.30 \mathrm{e}-8$ & 4.00 & $1.49 \mathrm{e}-5$ & 3.01 & $1.68 \mathrm{e}-7$ & 4.00 \\
\hline
\end{tabular}

TABLE 4. Convergence of $\left|\lambda_{h}-\tilde{\lambda}_{h}\right|$ to 0 for $\tau=1$.

\begin{tabular}{|c|c||cc|cc|cc|cc|}
\hline $\begin{array}{c}\text { degree } \\
k\end{array}$ & mesh & \multicolumn{2}{c|}{ first mode } & \multicolumn{2}{c|}{ second mode } & \multicolumn{2}{c|}{ fourth mode } & \multicolumn{2}{c|}{ sixth mode } \\
& $\ell$ & error & order & error & order & error & order & error & order \\
\hline \multirow{3}{*}{0} & 0 & $6.29 \mathrm{e}-1$ & -- & $2.83 \mathrm{e}-0$ & -- & $6.65 \mathrm{e}-0$ & -- & $8.95 \mathrm{e}-0$ & -- \\
& 1 & $2.70 \mathrm{e}-1$ & 1.22 & $1.40 \mathrm{e}-0$ & 1.01 & $3.36 \mathrm{e}-0$ & 0.99 & $4.72 \mathrm{e}-0$ & 0.92 \\
& 2 & $1.25 \mathrm{e}-1$ & 1.11 & $7.07 \mathrm{e}-1$ & 0.99 & $1.72 \mathrm{e}-0$ & 0.96 & $2.54 \mathrm{e}-0$ & 0.89 \\
& 3 & $5.99 \mathrm{e}-2$ & 1.06 & $3.56 \mathrm{e}-1$ & 0.99 & $8.85 \mathrm{e}-1$ & 0.96 & $1.34 \mathrm{e}-0$ & 0.92 \\
& 4 & $2.93 \mathrm{e}-2$ & 1.03 & $1.79 \mathrm{e}-1$ & 0.99 & $4.50 \mathrm{e}-1$ & 0.97 & $6.94 \mathrm{e}-1$ & 0.95 \\
\hline \multirow{4}{*}{1} & 0 & $6.21 \mathrm{e}-2$ & -- & $5.26 \mathrm{e}-1$ & -- & $1.85 \mathrm{e}-0$ & -- & $3.33 \mathrm{e}-0$ & -- \\
& 1 & $1.41 \mathrm{e}-2$ & 2.13 & $1.05 \mathrm{e}-1$ & 2.33 & $3.38 \mathrm{e}-1$ & 2.45 & $6.01 \mathrm{e}-1$ & 2.47 \\
& 2 & $3.37 \mathrm{e}-3$ & 2.07 & $2.30 \mathrm{e}-2$ & 2.19 & $6.70 \mathrm{e}-2$ & 2.33 & $1.12 \mathrm{e}-1$ & 2.42 \\
& 3 & $8.23 \mathrm{e}-4$ & 2.03 & $5.37 \mathrm{e}-3$ & 2.10 & $1.47 \mathrm{e}-2$ & 2.19 & $2.39 \mathrm{e}-2$ & 2.24 \\
& 4 & $2.03 \mathrm{e}-4$ & 2.02 & $1.30 \mathrm{e}-3$ & 2.05 & $3.44 \mathrm{e}-3$ & 2.10 & $5.48 \mathrm{e}-3$ & 2.12 \\
\hline \multirow{4}{*}{2} & 0 & $3.23 \mathrm{e}-2$ & -- & $2.09 \mathrm{e}-1$ & -- & $5.86 \mathrm{e}-1$ & -- & $9.78 \mathrm{e}-1$ & -- \\
& 1 & $8.11 \mathrm{e}-3$ & 1.99 & $5.10 \mathrm{e}-2$ & 2.03 & $1.32 \mathrm{e}-1$ & 2.15 & $2.09 \mathrm{e}-1$ & 2.23 \\
& 2 & $2.04 \mathrm{e}-3$ & 1.99 & $1.28 \mathrm{e}-2$ & 2.00 & $3.28 \mathrm{e}-2$ & 2.02 & $5.13 \mathrm{e}-2$ & 2.03 \\
& 3 & $5.12 \mathrm{e}-4$ & 1.99 & $3.20 \mathrm{e}-3$ & 2.00 & $8.20 \mathrm{e}-3$ & 2.00 & $1.28 \mathrm{e}-2$ & 2.00 \\
& 4 & $1.28 \mathrm{e}-4$ & 2.00 & $8.02 \mathrm{e}-4$ & 2.00 & $2.05 \mathrm{e}-3$ & 2.00 & $3.21 \mathrm{e}-3$ & 2.00 \\
\hline
\end{tabular}

convergence rate beyond the $O\left(h^{2 k+2}\right)$-rate was obtained. To conclude the discussion on the postprocessing, see Table 3, where the error and order of convergence of the approximate and postprocessed eigenfunctions is presented. We see that the convergence rate of the approximate eigenfunctions is $O\left(h^{k+1}\right)$, while the convergence rate of the postprocessed eigenfunctions is $O\left(h^{k+2}\right)$ for $k \geq 1$ and $O\left(h^{k+1}\right)$ for $k=0$. These results illustrate that the local postprocessing is effective for $k \geq 1$ as it increases the convergence rate of both the eigenvalue and eigenfunction approximations by one order.

In Table 4, we display the absolute value of difference between the approximate eigenvalues $\lambda_{h}$ and the perturbed eigenvalues $\tilde{\lambda}_{h}$ of Section 5 , namely $\left|\lambda_{h}-\tilde{\lambda}_{h}\right|$. We see that the numbers $\tilde{\lambda}_{h}$ can serve as good approximations of $\lambda_{h}$. The difference $\left|\lambda_{h}-\tilde{\lambda}_{h}\right|$ is seen to decrease with $h$ at the rate $O(h)$ for $k=0$. This is in accordance 
TABLE 5. Convergence of the approximate eigenvalues $\lambda_{h}$ for $\tau=h$.

\begin{tabular}{|c|c||cc|cc|cc|cc|}
\hline degree & mesh & \multicolumn{2}{c|}{ first mode } & \multicolumn{2}{c|}{ second mode } & \multicolumn{2}{c|}{ fourth mode } & \multicolumn{2}{c|}{ sixth mode } \\
$k$ & $\ell$ & error & order & error & order & error & order & error & order \\
\hline \multirow{4}{*}{0} & 0 & $1.42 \mathrm{e}-1$ & -- & $1.72 \mathrm{e}-0$ & -- & $3.76 \mathrm{e}-0$ & -- & $5.45 \mathrm{e}-0$ & -- \\
& 1 & $3.74 \mathrm{e}-1$ & -1.39 & $2.01 \mathrm{e}-0$ & -0.22 & $4.15 \mathrm{e}-0$ & -0.14 & $5.82 \mathrm{e}-0$ & -0.09 \\
& 2 & $4.33 \mathrm{e}-1$ & -0.21 & $2.09 \mathrm{e}-0$ & -0.05 & $4.27 \mathrm{e}-0$ & -0.04 & $5.91 \mathrm{e}-0$ & -0.02 \\
& 3 & $4.48 \mathrm{e}-1$ & -0.05 & $2.11 \mathrm{e}-0$ & -0.01 & $4.30 \mathrm{e}-0$ & -0.01 & $5.93 \mathrm{e}-0$ & -0.01 \\
& 4 & $4.52 \mathrm{e}-1$ & -0.01 & $2.11 \mathrm{e}-0$ & 0.00 & $4.31 \mathrm{e}-0$ & 0.00 & $5.94 \mathrm{e}-0$ & -0.00 \\
\hline \multirow{4}{*}{1} & 0 & $1.29 \mathrm{e}-2$ & -- & $2.45 \mathrm{e}-1$ & -- & $1.31 \mathrm{e}-0$ & -- & $2.47 \mathrm{e}-0$ & -- \\
& 1 & $4.44 \mathrm{e}-3$ & 1.54 & $9.15 \mathrm{e}-2$ & 1.42 & $5.57 \mathrm{e}-1$ & 1.23 & $1.24 \mathrm{e}-0$ & 0.99 \\
& 2 & $1.38 \mathrm{e}-3$ & 1.69 & $2.55 \mathrm{e}-2$ & 1.84 & $1.76 \mathrm{e}-1$ & 1.66 & $4.64 \mathrm{e}-1$ & 1.42 \\
& 3 & $3.62 \mathrm{e}-4$ & 1.93 & $6.56 \mathrm{e}-3$ & 1.96 & $4.73 \mathrm{e}-2$ & 1.90 & $1.37 \mathrm{e}-1$ & 1.76 \\
& 4 & $9.15 \mathrm{e}-5$ & 1.98 & $1.65 \mathrm{e}-3$ & 1.99 & $1.20 \mathrm{e}-2$ & 1.97 & $3.60 \mathrm{e}-2$ & 1.93 \\
\hline \multirow{4}{*}{2} & 0 & $1.81 \mathrm{e}-4$ & -- & $6.85 \mathrm{e}-3$ & -- & $7.60 \mathrm{e}-2$ & -- & $2.19 \mathrm{e}-1$ & -- \\
& 1 & $1.57 \mathrm{e}-5$ & 3.53 & $6.21 \mathrm{e}-4$ & 3.46 & $7.18 \mathrm{e}-3$ & 3.40 & $1.89 \mathrm{e}-2$ & 3.54 \\
& 2 & $1.25 \mathrm{e}-6$ & 3.65 & $4.24 \mathrm{e}-5$ & 3.87 & $4.93 \mathrm{e}-4$ & 3.87 & $1.26 \mathrm{e}-3$ & 3.90 \\
& 3 & $8.23 \mathrm{e}-8$ & 3.92 & $2.70 \mathrm{e}-6$ & 3.97 & $3.15 \mathrm{e}-5$ & 3.97 & $8.01 \mathrm{e}-5$ & 3.98 \\
& 4 & $5.21 \mathrm{e}-9$ & 3.98 & $1.70 \mathrm{e}-7$ & 3.99 & $1.98 \mathrm{e}-6$ & 3.99 & $5.02 \mathrm{e}-6$ & 3.99 \\
\hline
\end{tabular}

TABLE 6. Convergence of the approximate eigenvalues $\lambda_{h}$ for $\tau=1 / h$.

\begin{tabular}{|c|c||cc|cc|cc|cc|}
\hline degree & mesh & \multicolumn{2}{c|}{ first mode } & \multicolumn{2}{c|}{ second mode } & \multicolumn{2}{c|}{ fourth mode } & \multicolumn{2}{c|}{ sixth mode } \\
$k$ & $\ell$ & error & order & error & order & error & order & error & order \\
\hline \multirow{4}{*}{0} & 0 & $2.53 \mathrm{e}-1$ & -- & $7.42 \mathrm{e}-1$ & -- & $2.19 \mathrm{e}-0$ & -- & $3.57 \mathrm{e}-0$ & -- \\
& 1 & $5.73 \mathrm{e}-1$ & -1.18 & $7.76 \mathrm{e}-1$ & -0.07 & $8.88 \mathrm{e}-1$ & 1.30 & $6.80 \mathrm{e}-1$ & 2.39 \\
& 2 & $6.68 \mathrm{e}-1$ & -0.22 & $1.33 \mathrm{e}-0$ & -0.78 & $2.18 \mathrm{e}-0$ & -1.29 & $2.75 \mathrm{e}-0$ & -2.01 \\
& 3 & $6.92 \mathrm{e}-1$ & -0.05 & $1.49 \mathrm{e}-0$ & -0.16 & $2.56 \mathrm{e}-0$ & -0.23 & $3.39 \mathrm{e}-0$ & -0.30 \\
& 4 & $6.99 \mathrm{e}-1$ & -0.01 & $1.53 \mathrm{e}-0$ & -0.04 & $2.66 \mathrm{e}-0$ & -0.05 & $3.56 \mathrm{e}-0$ & -0.07 \\
\hline \multirow{4}{*}{1} & 0 & $1.46 \mathrm{e}-2$ & -- & $5.70 \mathrm{e}-2$ & -- & $3.88 \mathrm{e}-1$ & -- & $2.92 \mathrm{e}-1$ & -- \\
& 1 & $5.84 \mathrm{e}-3$ & 1.32 & $1.95 \mathrm{e}-2$ & 1.55 & $5.78 \mathrm{e}-2$ & 2.75 & $9.51 \mathrm{e}-2$ & 1.62 \\
& 2 & $1.59 \mathrm{e}-3$ & 1.88 & $6.75 \mathrm{e}-3$ & 1.53 & $2.33 \mathrm{e}-2$ & 1.31 & $3.05 \mathrm{e}-2$ & 1.64 \\
& 3 & $4.06 \mathrm{e}-4$ & 1.97 & $1.80 \mathrm{e}-3$ & 1.91 & $6.36 \mathrm{e}-3$ & 1.87 & $8.70 \mathrm{e}-3$ & 1.81 \\
& 4 & $1.02 \mathrm{e}-4$ & 1.99 & $4.57 \mathrm{e}-4$ & 1.98 & $1.62 \mathrm{e}-3$ & 1.97 & $2.24 \mathrm{e}-3$ & 1.96 \\
\hline \multirow{4}{*}{2} & 0 & $2.78 \mathrm{e}-4$ & -- & $1.12 \mathrm{e}-3$ & -- & $1.76 \mathrm{e}-2$ & -- & $2.47 \mathrm{e}-2$ & -- \\
& 1 & $2.55 \mathrm{e}-5$ & 3.45 & $1.82 \mathrm{e}-4$ & 2.62 & $1.11 \mathrm{e}-3$ & 3.99 & $2.10 \mathrm{e}-3$ & 3.57 \\
& 2 & $1.72 \mathrm{e}-6$ & 3.89 & $1.51 \mathrm{e}-5$ & 3.59 & $1.02 \mathrm{e}-4$ & 3.44 & $1.42 \mathrm{e}-4$ & 3.87 \\
& 3 & $1.10 \mathrm{e}-7$ & 3.97 & $1.00 \mathrm{e}-6$ & 3.91 & $6.89 \mathrm{e}-6$ & 3.89 & $9.97 \mathrm{e}-6$ & 3.83 \\
& 4 & $6.88 \mathrm{e}-9$ & 3.99 & $6.34 \mathrm{e}-8$ & 3.98 & $4.39 \mathrm{e}-7$ & 3.97 & $6.40 \mathrm{e}-7$ & 3.96 \\
\hline
\end{tabular}

with the estimate of Theorem $[5.3$. However, the same difference decreases at the rate $O\left(h^{2}\right)$ for $k \geq 1$, which is one order higher than that predicted by Theorem 5.3 . These results show that by solving a standard generalized eigenproblem for $\tilde{\lambda}_{h}$, we obtain very effective initial iterates for nonlinear iterative algorithms to solve the condensed nonlinear eigenproblem for $\lambda_{h}$. At the same time, we note that $\tilde{\lambda}_{h}$ by itself does not converge to the exact eigenvalue $\lambda$ at as fast a rate as $\lambda_{h}$.

Next, we examine the performance of the HDG method for different choices of $\tau$. In particular, we show the error and order of convergence of the approximate eigenvalues for $\tau=h$ in Table 5and for $\tau=1 / h$ in Table 6. We observe in both cases that the approximate eigenvalues converge at the rate $O\left(h^{2 k}\right)$. This convergence rate is one order less than the convergence rate of the approximate eigenvalues for $\tau=1$. A theoretical explanation for this phenomena follows from (11): When either $\tau=h$ or $\tau=1 / h$, one of the bounds in (11) deteriorate by one order in $h$. When 
TABLE 7. Convergence of the approximate $\left(\lambda_{h}\right)$ and postprocessed $\left(\lambda_{h}^{*}\right)$ eigenvalues for the L-shaped domain problem.

\begin{tabular}{|c|c||cc|cc||cc|cc|}
\hline \multicolumn{2}{|c||}{ eigenmode } & \multicolumn{6}{c||}{ first } & \multicolumn{4}{c|}{ third } \\
\hline $\begin{array}{c}\text { degree } \\
k\end{array}$ & mesh & \multicolumn{2}{|c||}{$\left|\lambda-\lambda_{h}\right|$} & \multicolumn{2}{|c|}{$\left|\lambda-\lambda_{h}^{*}\right|$} & \multicolumn{2}{|c|}{$\left|\lambda-\lambda_{h}\right|$} & \multicolumn{2}{|c}{$\left|\lambda-\lambda_{h}^{*}\right|$} \\
error & order & error & order & error & order & error & order \\
\hline \multirow{4}{*}{0} & 0 & $3.48 \mathrm{e}-0$ & -- & $4.20 \mathrm{e}-0$ & -- & $1.06 \mathrm{e}-9$ & -- & $1.12 \mathrm{e}-9$ & -- \\
& 1 & $2.12 \mathrm{e}-0$ & 0.72 & $2.71 \mathrm{e}-0$ & 0.63 & $7.39 \mathrm{e}-0$ & 0.52 & $8.10 \mathrm{e}-0$ & 0.46 \\
& 2 & $1.18 \mathrm{e}-0$ & 0.84 & $1.56 \mathrm{e}-0$ & 0.80 & $4.64 \mathrm{e}-0$ & 0.67 & $5.21 \mathrm{e}-0$ & 0.64 \\
& 3 & $6.24 \mathrm{e}-1$ & 0.92 & $8.34 \mathrm{e}-1$ & 0.90 & $2.66 \mathrm{e}-0$ & 0.80 & $3.02 \mathrm{e}-0$ & 0.79 \\
& 4 & $3.19 \mathrm{e}-1$ & 0.97 & $4.29 \mathrm{e}-1$ & 0.96 & $1.44 \mathrm{e}-0$ & 0.89 & $1.64 \mathrm{e}-0$ & 0.88 \\
\hline \multirow{4}{*}{1} & 0 & $5.04 \mathrm{e}-1$ & -- & $1.24 \mathrm{e}-1$ & -- & $4.04 \mathrm{e}-0$ & -- & $7.98 \mathrm{e}-1$ & -- \\
& 1 & $1.02 \mathrm{e}-1$ & 2.31 & $5.93 \mathrm{e}-2$ & 1.07 & $5.68 \mathrm{e}-1$ & 2.83 & $4.43 \mathrm{e}-2$ & 4.17 \\
& 2 & $2.82 \mathrm{e}-2$ & 1.85 & $2.36 \mathrm{e}-2$ & 1.33 & $6.19 \mathrm{e}-2$ & 3.20 & $2.35 \mathrm{e}-3$ & 4.24 \\
& 3 & $9.85 \mathrm{e}-3$ & 1.52 & $9.37 \mathrm{e}-3$ & 1.33 & $7.10 \mathrm{e}-3$ & 3.12 & $1.39 \mathrm{e}-4$ & 4.08 \\
& 4 & $3.77 \mathrm{e}-3$ & 1.39 & $3.73 \mathrm{e}-3$ & 1.33 & $8.50 \mathrm{e}-4$ & 3.06 & $8.15 \mathrm{e}-6$ & 4.09 \\
\hline \multirow{4}{*}{2} & 0 & $6.93 \mathrm{e}-2$ & -- & $5.78 \mathrm{e}-2$ & -- & $3.03 \mathrm{e}-1$ & -- & $3.91 \mathrm{e}-2$ & -- \\
& 1 & $2.35 \mathrm{e}-2$ & 1.56 & $2.36 \mathrm{e}-2$ & 1.29 & $7.73 \mathrm{e}-3$ & 5.29 & $3.48 \mathrm{e}-4$ & 6.81 \\
& 2 & $9.32 \mathrm{e}-3$ & 1.33 & $9.41 \mathrm{e}-3$ & 1.33 & $2.19 \mathrm{e}-4$ & 5.14 & $4.48 \mathrm{e}-6$ & 6.28 \\
& 3 & $3.73 \mathrm{e}-3$ & 1.32 & $3.75 \mathrm{e}-3$ & 1.33 & $6.50 \mathrm{e}-6$ & 5.07 & $6.13 \mathrm{e}-8$ & 6.19 \\
& 4 & $1.49 \mathrm{e}-3$ & 1.33 & $1.49 \mathrm{e}-3$ & 1.33 & $1.98 \mathrm{e}-7$ & 5.04 & $7.9 \mathrm{e}-10$ & 6.28 \\
\hline
\end{tabular}

these revised estimates are used in the ensuing eigenvalue convergence analysis, we obtain the reduced $O\left(h^{2 k}\right)$-rate.

6.3. L-shaped domain. To study the limitations imposed by singularities of eigenfunctions, we consider the L-shaped domain $\Omega=\Omega_{0} \backslash \Omega_{1}$, where $\Omega_{0} \equiv(0,2) \times(0,2)$ and $\Omega_{1} \equiv(1,2) \times(1,2)$ are the square domains. This domain has both singular and smooth eigenfunctions, so offers an interesting example to study the changes in convergence rates due to singularities. As before, we consider triangular meshes that are successive uniform refinements of an initial uniform mesh. The initial mesh is obtained as in the previous example using a $4 \times 4$ uniform grid of $\Omega_{0}$, except we now omit all triangles in $\Omega_{1}$.

Since $\Omega$ has a reentrant corner at the point $(1,1)$, some eigenfunctions are singular. Specifically, we may only expect Theorem 4.1 to hold with $s_{\lambda}=\frac{2}{3}-\varepsilon$ for an arbitrarily small $\varepsilon>0$ for singular eigenfunctions. For this L-shaped domain the first eigenmode is singular and the corresponding eigenvalue is calculated in 4 . as $\lambda_{1}=9.63972384464540$. It is interesting to note that the third eigenmode is smooth and the third eigenvalue is known exactly as $\lambda_{3}=2 \pi^{2}$.

The errors and resulting order of convergence for the approximate and postprocessed eigenvalues are reported in Table 7 for the first and third eigenmodes. We observe that the convergence rate of the approximate smallest eigenvalue is at most $O\left(h^{4 / 3}\right)$ which agrees with the a priori error estimate given by Theorem 4.1. Furthermore, the postprocessed smallest eigenvalue also converges at the same order $4 / 3$ for $k \geq 1$. However, for the third eigenmode, the approximate eigenvalue converges at order $O\left(h^{2 k+1}\right)$ and the postprocessed eigenvalue converges at order $O\left(h^{2 k+2}\right)$ for $k \geq 1$.

6.4. Other polynomial spaces. So far the presentation focused on the case when the same polynomial degree $k$ is employed to approximate the solution, the flux, and the trace. It is interesting to examine the case of mixed degrees. Since $M_{h}$ determines the size of the global system, let us hold $M_{h}$ fixed as set in (6) consisting 
TABLE 8. Summary of convergence rates for smooth eigenfunctions

\begin{tabular}{|l||ccccc|}
\hline \multicolumn{1}{|c|}{ Method } & \multicolumn{5}{c|}{ Convergence rates } \\
\cline { 2 - 6 } & $\lambda_{h}$ & $\lambda_{h}^{*}$ & $\vec{q}_{h}$ & $u_{h}$ & $u_{h}^{*}$ \\
\hline HDG, equal degree $k \geq 0, \tau=1$ & $2 k+1$ & $2 k+2$ & $k+1$ & $k+1$ & $k+2-\delta_{k 0}$ \\
HDG, equal degree $k \geq 1, \tau=h$ & $2 k$ & $2 k+2$ & $k+1$ & $k$ & $k+2$ \\
HDG, equal degree $k \geq 1, \tau=1 / h$ & $2 k$ & $2 k$ & $k$ & $k+1$ & $k+1$ \\
HDG, Case $1, k \geq 1, \tau=1$ & $2 k-1$ & $2 k$ & $k$ & $k$ & $k+1$ \\
HDG, Case $2, k \geq 1, \tau=1$ & $2 k-1$ & $2 k-\delta_{k 1}$ & $k$ & $k$ & $k+1$ \\
Interior penalty DG [2], $k \geq 1$ & $2 k$ & - & - & $k+1$ & - \\
HRT [9] $(k \geq 0, \tau=0)$ & $2 k+2$ & $2 k+2$ & $k+1$ & $k+1$ & $k+2$ \\
BDM (Case $1, k \geq 1, \tau=0)$ & $2 k$ & $2 k+2$ & $k+1$ & $k$ & $k+2$ \\
\hline
\end{tabular}

of functions of degree $k$ on the element interfaces, while varying the degrees of $W_{h}$ and $V_{h}$ as follows:

Case 1. $[\tau]_{K} \geq 0$ on $\partial K$ for all $K \in \mathscr{T}_{h}, k \geq 1$, and

$$
\begin{aligned}
W_{h} & =\left\{w: \text { for every mesh element } K,\left.w\right|_{K} \in P_{k-1}(K)\right\}, \\
V_{h} & =\left\{\vec{v}: \text { for every mesh element } K,\left.\vec{v}\right|_{K} \in P_{k}(K)^{n}\right\} .
\end{aligned}
$$

Case 2. $[\tau]_{K}>0$ on $\partial K$ for all $K \in \mathscr{T}_{h}, k \geq 1$, and

$$
\begin{aligned}
W_{h} & =\left\{w: \text { for every mesh element } K,\left.w\right|_{K} \in P_{k}(K)\right\}, \\
V_{h} & =\left\{\vec{v}: \text { for every mesh element } K,\left.\vec{v}\right|_{K} \in P_{k-1}(K)^{n}\right\} .
\end{aligned}
$$

These cases are interesting because the source problem (7) is uniquely solvable 8 , see eq. (3.5) and Proposition 3.2]. One can now follow the same techniques in [7, Lemma 3.2 and Theorem 3.1] to obtain a bound for the norm of the operator $\mathcal{U}_{W}$, leading to results analogous to Theorem 5.3 for the reduced eigenproblem in both of these cases. On the other hand, rigorous proofs of eigenvalue convergence rates for general $\tau$ are yet to be developed for these cases: Ingredients (of Section 4) that need generalization include the projection satisfying (11) and the $\tau$-explicit estimates (12)-(13).

Nevertheless, numerical results are not encouraging in these cases. Returning to the eigenproblem on the square described in 6.2 , the convergence of approximate and postprocessed eigenvalues in both cases, obtained with $\tau=1$, are presented in Tables 9 12. We observe that the approximate eigenvalues converge at the rate $O\left(h^{2 k-1}\right)$ in both cases. It also appears that the postprocessed eigenvalues converge at the rate $O\left(h^{2 k}\right)$, except in Case 2 with $k=1$, where $\lambda_{h}^{*}$ converges at the same rate as $\lambda_{h}$. Comparing the results from approximation spaces of mixed and equal degree, we find that the equal-degree spaces give two orders faster convergence rate, so is clearly preferable in the eigenvalue context.

The special case of $\tau \equiv 0$ deserves further remarks. In this case, if $V_{h}$ is changed to the (larger) piecewise Raviart-Thomas space, then the resulting HDG eigenproblem is the same as the hybridized Raviart-Thomas eigenproblem. This case is fully studied in 9]. Next, consider the special case of $\tau \equiv 0$ in Case 1. Then, the HDG formulation reduces to the (hybridized) mixed Brezzi-Douglas-Marini (BDM) eigenproblem, for which a complete convergence theory is available in 5. In particular, it follows from their results that the BDM eigenvalue convergence rate is $O\left(h^{2 k}\right)$. However, the postprocessing of 6.1 provides a way to make the BDM eigenvalues 
more competitive: We observe, in Table 13, that postprocessed BDM eigenvalues converge at $O\left(h^{2 k+2}\right)$, the rate also observed for the HRT and HDG eigenvalues after the same postprocessing.

We conclude with a summary of the convergence rates in Table 8. Its entries are based on the observed and known convergence rates in various cases for the first eigenpair of the square domain example in 86.2 . Note that, as before, the convergence of all functions is measured in the $L^{2}(\Omega)$-norm.

TABLE 9. Convergence of the approximate eigenvalues $\lambda_{h}$ for (the mixed-degree) Case 1 with $\tau=1$.

\begin{tabular}{|c|c||cc|cc|cc|cc|}
\hline degree & mesh & \multicolumn{2}{|c|}{ first mode } & \multicolumn{2}{c|}{ second mode } & \multicolumn{2}{c|}{ fourth mode } & \multicolumn{2}{c|}{ sixth mode } \\
$k$ & $\ell$ & error & order & error & order & error & order & error & order \\
\hline \multirow{4}{*}{1} & 0 & $1.12 \mathrm{e}-0$ & -- & $2.68 \mathrm{e}-0$ & -- & $4.90 \mathrm{e}-0$ & -- & $7.05 \mathrm{e}-0$ & -- \\
& 1 & $5.78 \mathrm{e}-1$ & 0.96 & $1.33 \mathrm{e}-0$ & 1.01 & $2.50 \mathrm{e}-0$ & 0.97 & $3.64 \mathrm{e}-0$ & 0.95 \\
& 2 & $2.93 \mathrm{e}-1$ & 0.98 & $6.53 \mathrm{e}-1$ & 1.03 & $1.23 \mathrm{e}-0$ & 1.03 & $1.67 \mathrm{e}-0$ & 1.12 \\
& 3 & $1.48 \mathrm{e}-1$ & 0.99 & $3.23 \mathrm{e}-1$ & 1.02 & $6.05 \mathrm{e}-1$ & 1.02 & $7.91 \mathrm{e}-1$ & 1.08 \\
& 4 & $7.41 \mathrm{e}-2$ & 0.99 & $1.60 \mathrm{e}-1$ & 1.01 & $3.00 \mathrm{e}-1$ & 1.01 & $3.84 \mathrm{e}-1$ & 1.04 \\
\hline \multirow{4}{*}{2} & 0 & $3.41 \mathrm{e}-2$ & -- & $1.54 \mathrm{e}-1$ & -- & $5.39 \mathrm{e}-1$ & -- & $8.13 \mathrm{e}-1$ & -- \\
& 1 & $4.72 \mathrm{e}-3$ & 2.85 & $2.13 \mathrm{e}-2$ & 2.85 & $7.93 \mathrm{e}-2$ & 2.76 & $1.12 \mathrm{e}-1$ & 2.85 \\
& 2 & $6.18 \mathrm{e}-4$ & 2.93 & $2.76 \mathrm{e}-3$ & 2.95 & $1.03 \mathrm{e}-2$ & 2.94 & $1.43 \mathrm{e}-2$ & 2.98 \\
& 3 & $7.90 \mathrm{e}-5$ & 2.97 & $3.49 \mathrm{e}-4$ & 2.98 & $1.30 \mathrm{e}-3$ & 2.99 & $1.78 \mathrm{e}-3$ & 3.00 \\
& 4 & $9.99 \mathrm{e}-6$ & 2.98 & $4.38 \mathrm{e}-5$ & 2.99 & $1.62 \mathrm{e}-4$ & 3.00 & $2.22 \mathrm{e}-4$ & 3.01 \\
\hline \multirow{4}{*}{3} & 0 & $5.81 \mathrm{e}-4$ & -- & $4.88 \mathrm{e}-3$ & -- & $3.56 \mathrm{e}-2$ & -- & $5.23 \mathrm{e}-2$ & -- \\
& 1 & $1.99 \mathrm{e}-5$ & 4.87 & $1.76 \mathrm{e}-4$ & 4.80 & $1.31 \mathrm{e}-3$ & 4.76 & $1.87 \mathrm{e}-3$ & 4.80 \\
& 2 & $6.44 \mathrm{e}-7$ & 4.95 & $5.68 \mathrm{e}-6$ & 4.95 & $4.25 \mathrm{e}-5$ & 4.95 & $5.97 \mathrm{e}-5$ & 4.97 \\
& 3 & $2.04 \mathrm{e}-8$ & 4.98 & $1.79 \mathrm{e}-7$ & 4.99 & $1.33 \mathrm{e}-6$ & 4.99 & $1.86 \mathrm{e}-6$ & 5.00 \\
& 4 & $6.43 \mathrm{e}-10$ & 4.99 & $1.13 \mathrm{e}-8$ & 3.99 & $4.16 \mathrm{e}-8$ & 5.00 & $5.78 \mathrm{e}-8$ & 5.01 \\
\hline
\end{tabular}

TABLE 10. Convergence of the postprocessed eigenvalues $\lambda_{h}^{*}$ for (the mixed-degree) Case 1 with $\tau=1$.

\begin{tabular}{|c|c||cc|cc|cc|cc|}
\hline degree & mesh & \multicolumn{2}{|c|}{ first mode } & \multicolumn{2}{c|}{ second mode } & \multicolumn{2}{c|}{ fourth mode } & \multicolumn{2}{c|}{ sixth mode } \\
$k$ & $\ell$ & error & order & error & order & error & order & error & order \\
\hline \multirow{4}{*}{1} & 0 & $1.20 \mathrm{e}-1$ & -- & $3.54 \mathrm{e}-1$ & -- & $7.98 \mathrm{e}-1$ & -- & $1.06 \mathrm{e}-0$ & -- \\
& 1 & $3.74 \mathrm{e}-2$ & 1.68 & $9.45 \mathrm{e}-2$ & 1.90 & $2.57 \mathrm{e}-1$ & 1.64 & $1.98 \mathrm{e}-1$ & 2.43 \\
& 2 & $1.07 \mathrm{e}-2$ & 1.81 & $2.51 \mathrm{e}-2$ & 1.91 & $7.70 \mathrm{e}-2$ & 1.74 & $3.22 \mathrm{e}-2$ & 2.62 \\
& 3 & $2.88 \mathrm{e}-3$ & 1.89 & $6.55 \mathrm{e}-3$ & 1.94 & $2.11 \mathrm{e}-2$ & 1.86 & $6.26 \mathrm{e}-3$ & 2.36 \\
& 4 & $7.51 \mathrm{e}-4$ & 1.94 & $1.68 \mathrm{e}-3$ & 1.96 & $5.52 \mathrm{e}-3$ & 1.94 & $1.39 \mathrm{e}-3$ & 2.17 \\
\hline \multirow{4}{*}{2} & 0 & $5.98 \mathrm{e}-3$ & -- & $2.80 \mathrm{e}-2$ & -- & $1.13 \mathrm{e}-1$ & -- & $1.50 \mathrm{e}-1$ & -- \\
& 1 & $4.86 \mathrm{e}-4$ & 3.62 & $2.22 \mathrm{e}-3$ & 3.65 & $8.95 \mathrm{e}-3$ & 3.66 & $1.21 \mathrm{e}-2$ & 3.63 \\
& 2 & $3.46 \mathrm{e}-5$ & 3.81 & $1.54 \mathrm{e}-4$ & 3.85 & $6.03 \mathrm{e}-4$ & 3.89 & $8.41 \mathrm{e}-4$ & 3.85 \\
& 3 & $2.31 \mathrm{e}-6$ & 3.91 & $1.00 \mathrm{e}-5$ & 3.94 & $3.87 \mathrm{e}-5$ & 3.96 & $5.40 \mathrm{e}-5$ & 3.96 \\
& 4 & $1.49 \mathrm{e}-7$ & 3.95 & $6.39 \mathrm{e}-7$ & 3.97 & $2.44 \mathrm{e}-6$ & 3.99 & $3.39 \mathrm{e}-6$ & 3.99 \\
\hline \multirow{4}{*}{3} & 0 & $7.95 \mathrm{e}-5$ & -- & $6.94 \mathrm{e}-4$ & -- & $6.15 \mathrm{e}-3$ & -- & $8.36 \mathrm{e}-3$ & -- \\
& 1 & $1.42 \mathrm{e}-6$ & 5.80 & $1.25 \mathrm{e}-5$ & 5.79 & $1.05 \mathrm{e}-4$ & 5.87 & $1.46 \mathrm{e}-4$ & 5.84 \\
& 2 & $2.37 \mathrm{e}-8$ & 5.91 & $2.04 \mathrm{e}-7$ & 5.94 & $1.64 \mathrm{e}-6$ & 6.00 & $2.24 \mathrm{e}-6$ & 6.02 \\
& 3 & $3.77 \mathrm{e}-10$ & 5.97 & $3.23 \mathrm{e}-9$ & 5.98 & $2.55 \mathrm{e}-8$ & 6.01 & $3.45 \mathrm{e}-8$ & 6.02 \\
& 4 & $6.08 \mathrm{e}-12$ & 5.95 & $5.04 \mathrm{e}-11$ & 6.00 & $3.77 \mathrm{e}-10$ & 6.08 & $5.14 \mathrm{e}-10$ & 6.07 \\
\hline
\end{tabular}


TABLE 11. Convergence of the approximate eigenvalues $\lambda_{h}$ for (the mixed-degree) Case 2 with $\tau=1$.

\begin{tabular}{|c|c||cc|cc|cc|cc|}
\hline degree & mesh & \multicolumn{2}{|c|}{ first mode } & \multicolumn{2}{c|}{ second mode } & \multicolumn{2}{c|}{ fourth mode } & \multicolumn{2}{c|}{ sixth mode } \\
$k$ & $\ell$ & error & order & error & order & error & order & error & order \\
\hline \multirow{4}{*}{1} & 0 & $3.89 \mathrm{e}-1$ & -- & $1.92 \mathrm{e}-0$ & -- & $3.88 \mathrm{e}-0$ & -- & $6.18 \mathrm{e}-0$ & -- \\
& 1 & $2.15 \mathrm{e}-1$ & 0.85 & $1.19 \mathrm{e}-0$ & 0.69 & $2.61 \mathrm{e}-0$ & 0.57 & $3.88 \mathrm{e}-0$ & 0.67 \\
& 2 & $1.12 \mathrm{e}-1$ & 0.94 & $6.60 \mathrm{e}-1$ & 0.85 & $1.54 \mathrm{e}-0$ & 0.76 & $2.35 \mathrm{e}-0$ & 0.72 \\
& 3 & $5.73 \mathrm{e}-2$ & 0.97 & $3.47 \mathrm{e}-1$ & 0.93 & $8.45 \mathrm{e}-1$ & 0.87 & $1.31 \mathrm{e}-0$ & 0.85 \\
& 4 & $2.89 \mathrm{e}-2$ & 0.99 & $1.78 \mathrm{e}-1$ & 0.96 & $4.44 \mathrm{e}-1$ & 0.93 & $6.89 \mathrm{e}-1$ & 0.92 \\
\hline \multirow{4}{*}{2} & 0 & $1.75 \mathrm{e}-2$ & -- & $3.33 \mathrm{e}-1$ & -- & $1.19 \mathrm{e}-0$ & -- & $2.18 \mathrm{e}-0$ & -- \\
& 1 & $2.19 \mathrm{e}-3$ & 3.00 & $4.28 \mathrm{e}-2$ & 2.96 & $1.64 \mathrm{e}-1$ & 2.86 & $3.39 \mathrm{e}-1$ & 2.68 \\
& 2 & $2.69 \mathrm{e}-4$ & 3.03 & $5.08 \mathrm{e}-3$ & 3.08 & $1.88 \mathrm{e}-2$ & 3.12 & $3.83 \mathrm{e}-2$ & 3.15 \\
& 3 & $3.32 \mathrm{e}-5$ & 3.02 & $6.12 \mathrm{e}-4$ & 3.05 & $2.23 \mathrm{e}-3$ & 3.08 & $4.44 \mathrm{e}-3$ & 3.11 \\
& 4 & $4.12 \mathrm{e}-6$ & 3.01 & $7.50 \mathrm{e}-5$ & 3.03 & $2.70 \mathrm{e}-4$ & 3.04 & $5.32 \mathrm{e}-4$ & 3.06 \\
\hline \multirow{4}{*}{3} & 0 & $2.78 \mathrm{e}-4$ & -- & $8.57 \mathrm{e}-3$ & -- & $8.97 \mathrm{e}-2$ & -- & $2.19 \mathrm{e}-1$ & -- \\
& 1 & $8.71 \mathrm{e}-6$ & 4.99 & $2.52 \mathrm{e}-4$ & 5.09 & $2.52 \mathrm{e}-3$ & 5.15 & $5.67 \mathrm{e}-3$ & 5.27 \\
& 2 & $2.70 \mathrm{e}-7$ & 5.01 & $7.54 \mathrm{e}-6$ & 5.06 & $7.36 \mathrm{e}-5$ & 5.10 & $1.60 \mathrm{e}-4$ & 5.15 \\
& 3 & $8.36 \mathrm{e}-9$ & 5.01 & $2.30 \mathrm{e}-7$ & 5.04 & $2.21 \mathrm{e}-6$ & 5.06 & $4.75 \mathrm{e}-6$ & 5.08 \\
& 4 & $2.6 \mathrm{e}-10$ & 5.01 & $7.08 \mathrm{e}-9$ & 5.02 & $6.77 \mathrm{e}-8$ & 5.03 & $1.44 \mathrm{e}-7$ & 5.04 \\
\hline
\end{tabular}

TABLE 12. Convergence of the postprocessed eigenvalues $\lambda_{h}^{*}$ for (the mixed-degree) Case 2 with $\tau=1$.

\begin{tabular}{|c|c||cc|cc|cc|cc|}
\hline degree & mesh & \multicolumn{2}{c|}{ first mode } & \multicolumn{2}{c|}{ second mode } & \multicolumn{2}{c|}{ fourth mode } & \multicolumn{2}{c|}{ sixth mode } \\
$k$ & $\ell$ & error & order & error & order & error & order & error & order \\
\hline \multirow{4}{*}{1} & 0 & $3.77 \mathrm{e}-1$ & -- & $1.87 \mathrm{e}-0$ & -- & $3.80 \mathrm{e}-0$ & -- & $6.24 \mathrm{e}-0$ & -- \\
& 1 & $2.13 \mathrm{e}-1$ & 0.83 & $1.17 \mathrm{e}-0$ & 0.68 & $2.56 \mathrm{e}-0$ & 0.57 & $3.83 \mathrm{e}-0$ & 0.70 \\
& 2 & $1.12 \mathrm{e}-1$ & 0.93 & $6.55 \mathrm{e}-1$ & 0.83 & $1.53 \mathrm{e}-0$ & 0.74 & $2.33 \mathrm{e}-0$ & 0.72 \\
& 3 & $5.72 \mathrm{e}-2$ & 0.97 & $3.46 \mathrm{e}-1$ & 0.92 & $8.42 \mathrm{e}-1$ & 0.86 & $1.30 \mathrm{e}-0$ & 0.84 \\
& 4 & $2.89 \mathrm{e}-2$ & 0.99 & $1.78 \mathrm{e}-1$ & 0.96 & $4.43 \mathrm{e}-1$ & 0.93 & $6.89 \mathrm{e}-1$ & 0.92 \\
\hline \multirow{4}{*}{2} & 0 & $2.26 \mathrm{e}-4$ & -- & $1.95 \mathrm{e}-3$ & -- & $1.01 \mathrm{e}-2$ & -- & $2.36 \mathrm{e}-2$ & -- \\
& 1 & $3.18 \mathrm{e}-5$ & 2.83 & $5.02 \mathrm{e}-4$ & 1.96 & $1.66 \mathrm{e}-3$ & 2.61 & $3.05 \mathrm{e}-3$ & 2.95 \\
& 2 & $2.58 \mathrm{e}-6$ & 3.62 & $4.48 \mathrm{e}-5$ & 3.48 & $1.34 \mathrm{e}-4$ & 3.63 & $2.15 \mathrm{e}-4$ & 3.83 \\
& 3 & $1.80 \mathrm{e}-7$ & 3.84 & $3.20 \mathrm{e}-6$ & 3.81 & $1.08 \mathrm{e}-5$ & 3.62 & $1.52 \mathrm{e}-5$ & 3.82 \\
& 4 & $1.18 \mathrm{e}-8$ & 3.93 & $2.12 \mathrm{e}-7$ & 3.92 & $7.40 \mathrm{e}-7$ & 3.87 & $1.01 \mathrm{e}-6$ & 3.92 \\
\hline \multirow{4}{*}{3} & 0 & $4.92 \mathrm{e}-6$ & -- & $1.20 \mathrm{e}-4$ & -- & $3.98 \mathrm{e}-3$ & -- & $1.11 \mathrm{e}-2$ & -- \\
& 1 & $4.93 \mathrm{e}-8$ & 6.64 & $7.34 \mathrm{e}-7$ & 7.35 & $1.77 \mathrm{e}-5$ & 7.82 & $7.32 \mathrm{e}-5$ & 7.25 \\
& 2 & $5.53 \mathrm{e}-10$ & 6.48 & $5.59 \mathrm{e}-9$ & 7.04 & $1.44 \mathrm{e}-7$ & 6.94 & $8.61 \mathrm{e}-7$ & 6.41 \\
& 3 & $5.63 \mathrm{e}-12$ & 6.62 & $4.44 \mathrm{e}-11$ & 6.97 & $1.69 \mathrm{e}-9$ & 6.41 & $1.25 \mathrm{e}-8$ & 6.11 \\
& 4 & $1.04 \mathrm{e}-13$ & 5.76 & $4.01 \mathrm{e}-13$ & 6.79 & $2.40 \mathrm{e}-11$ & 6.14 & $1.71 \mathrm{e}-10$ & 6.19 \\
\hline
\end{tabular}


TABLE 13. Convergence of the postprocessed BDM eigenvalues $\lambda_{h}^{*}$ obtained using the implementation of mixed-degree Case 1 with $\tau=0$.

\begin{tabular}{|c|c||cc|cc|cc|cc|}
\hline degree & \multicolumn{1}{|c||}{ mesh } & \multicolumn{2}{c|}{ first mode } & \multicolumn{2}{c|}{ second mode } & \multicolumn{2}{c|}{ fourth mode } & \multicolumn{2}{c|}{ sixth mode } \\
$k$ & $\ell$ & error & order & error & order & error & order & error & order \\
\hline \multirow{4}{*}{1} & 0 & $8.30 \mathrm{e}-4$ & -- & $2.40 \mathrm{e}-2$ & -- & $1.35 \mathrm{e}-1$ & -- & $2.32 \mathrm{e}-1$ & -- \\
& 1 & $3.33 \mathrm{e}-5$ & 4.64 & $1.17 \mathrm{e}-3$ & 4.35 & $9.96 \mathrm{e}-3$ & 3.76 & $1.47 \mathrm{e}-2$ & 3.98 \\
& 2 & $1.65 \mathrm{e}-6$ & 4.33 & $6.63 \mathrm{e}-5$ & 4.15 & $6.43 \mathrm{e}-4$ & 3.95 & $9.44 \mathrm{e}-4$ & 3.96 \\
& 3 & $9.31 \mathrm{e}-8$ & 4.15 & $3.98 \mathrm{e}-6$ & 4.06 & $4.02 \mathrm{e}-5$ & 4.00 & $5.92 \mathrm{e}-5$ & 4.00 \\
\hline \multirow{4}{*}{2} & 0 & $6.54 \mathrm{e}-5$ & -- & $1.37 \mathrm{e}-3$ & -- & $1.33 \mathrm{e}-2$ & -- & $2.03 \mathrm{e}-2$ & -- \\
& 1 & $1.12 \mathrm{e}-6$ & 5.86 & $2.62 \mathrm{e}-5$ & 5.71 & $2.71 \mathrm{e}-4$ & 5.62 & $4.76 \mathrm{e}-4$ & 5.41 \\
& 2 & $1.80 \mathrm{e}-8$ & 5.97 & $4.31 \mathrm{e}-7$ & 5.93 & $4.53 \mathrm{e}-6$ & 5.90 & $8.60 \mathrm{e}-6$ & 5.79 \\
& 3 & $2.82 \mathrm{e}-10$ & 5.99 & $6.82 \mathrm{e}-9$ & 5.98 & $7.21 \mathrm{e}-8$ & 5.98 & $1.39 \mathrm{e}-7$ & 5.95 \\
\hline \multirow{4}{*}{3} & 0 & $5.73 \mathrm{e}-7$ & -- & $1.97 \mathrm{e}-5$ & -- & $5.21 \mathrm{e}-4$ & -- & $7.91 \mathrm{e}-4$ & -- \\
& 1 & $2.32 \mathrm{e}-9$ & 7.95 & $2.30 \mathrm{e}-7$ & 6.42 & $2.33 \mathrm{e}-6$ & 7.80 & $3.66 \mathrm{e}-6$ & 7.75 \\
& 2 & $7.82 \mathrm{e}-12$ & 8.21 & $9.08 \mathrm{e}-10$ & 7.99 & $9.36 \mathrm{e}-9$ & 7.96 & $1.47 \mathrm{e}-8$ & 7.96 \\
& 3 & $3.12 \mathrm{e}-14$ & 7.97 & $1.89 \mathrm{e}-12$ & 8.91 & $3.15 \mathrm{e}-11$ & 8.22 & $5.24 \mathrm{e}-11$ & 8.13 \\
\hline
\end{tabular}

\section{REFERENCES}

[1] Philip M. Anselone, Collectively Compact Operator Approximation Theory and Applications to Integral Equations, Prentice-Hall Inc., Englewood Cliffs, N. J., 1971. With an appendix by Joel Davis; Prentice-Hall Series in Automatic Computation. MR0443383 (56 \#1753)

[2] Paola F. Antonietti, Annalisa Buffa, and Ilaria Perugia, Discontinuous Galerkin approximation of the Laplace eigenproblem, Comput. Methods Appl. Mech. Engrg. 195 (2006), no. 25-28, 3483-3503, DOI 10.1016/j.cma.2005.06.023. MR2220929 (2006m:65249)

[3] F. L. Bauer and C. T. Fike, Norms and exclusion theorems, Numer. Math. 2 (1960), 137-141. MR.0118729 (22 \#9500)

[4] Timo Betcke and Lloyd N. Trefethen, Reviving the method of particular solutions, SIAM Rev. 47 (2005), no. 3, 469-491 (electronic), DOI 10.1137/S0036144503437336. MR.2178637 (2006k:65344)

[5] Daniele Boffi, Franco Brezzi, and Lucia Gastaldi, On the problem of spurious eigenvalues in the approximation of linear elliptic problems in mixed form, Math. Comp. 69 (2000), no. 229, 121-140, DOI 10.1090/S0025-5718-99-01072-8. MR.1642801 (2000i:65175)

[6] J. H. Bramble and J. E. Osborn, Rate of convergence estimates for nonselfadjoint eigenvalue approximations, Math. Comp. 27 (1973), 525-549. MR0366029 (51 \#2280)

[7] B. Cockburn, O. Dubois, J. Gopalakrishnan, and S. Tan, Multigrid for an HDG method, IMA J. Numer. Anal. 34 (2014), no. 4, 1386-1425, DOI 10.1093/imanum/drt024. MR3269430

[8] Bernardo Cockburn, Jayadeep Gopalakrishnan, and Raytcho Lazarov, Unified hybridization of discontinuous Galerkin, mixed, and continuous Galerkin methods for second order elliptic problems, SIAM J. Numer. Anal. 47 (2009), no. 2, 1319-1365, DOI 10.1137/070706616. MR2485455(2010b:65251)

[9] B. Cockburn, J. Gopalakrishnan, F. Li, N.-C. Nguyen, and J. Peraire, Hybridization and postprocessing techniques for mixed eigenfunctions, SIAM J. Numer. Anal. 48 (2010), no. 3, 857-881, DOI 10.1137/090765894. MR2669393(2011f:65244)

[10] Bernardo Cockburn, Jayadeep Gopalakrishnan, and Francisco-Javier Sayas, A projectionbased error analysis of HDG methods, Math. Comp. 79 (2010), no. 271, 1351-1367, DOI 10.1090/S0025-5718-10-02334-3. MR.2629996(2011d:65354)

[11] Jean Descloux, Nabil Nassif, and Jacques Rappaz, On spectral approximation. I. The problem of convergence (English, with French summary), RAIRO Anal. Numér. 12 (1978), no. 2, 97112, iii. MR0483400(58 \#3404a) 
[12] Nelson Dunford and Jacob T. Schwartz, Linear operators. Part II. Spectral theory. Selfadjoint operators in Hilbert space; With the assistance of William G. Bade and Robert G. Bartle; Reprint of the 1963 original, Wiley Classics Library, John Wiley \& Sons Inc., New York, 1988; A Wiley-Interscience Publication. MR1009163 (90g:47001b)

[13] Stefano Giani and Edward J. C. Hall, An a posteriori error estimator for hp-adaptive discontinuous Galerkin methods for elliptic eigenvalue problems, Math. Models Methods Appl. Sci. 22 (2012), no. 10, 1250030, 35, DOI 10.1142/S0218202512500303. MR2974168

[14] Tosio Kato, Perturbation theory for nullity, deficiency and other quantities of linear operators, J. Analyse Math. 6 (1958), 261-322. MR0107819 (21 \#6541)

[15] — Perturbation Theory for Linear Operators, Classics in Mathematics, Springer-Verlag, Berlin, 1995. Reprint of the 1980 edition.

[16] B. Mercier, J. Osborn, J. Rappaz, and P.-A. Raviart, Eigenvalue approximation by mixed and hybrid methods, Math. Comp. 36 (1981), no. 154, 427-453, DOI 10.2307/2007651. MR606505 (82b:65108)

[17] John E. Osborn, Spectral approximation for compact operators, Math. Comput. 29 (1975), 712-725. MR0383117 (52 \#3998)

[18] Rolf Stenberg, Postprocessing schemes for some mixed finite elements (English, with French summary), RAIRO Modél. Math. Anal. Numér. 25 (1991), no. 1, 151-167. MR.1086845 (92a:65303)

[19] Hermann Weyl, Das asymptotische Verteilungsgesetz der Eigenwerte linearer partieller Differentialgleichungen (mit einer Anwendung auf die Theorie der Hohlraumstrahlung) (German), Math. Ann. 71 (1912), no. 4, 441-479, DOI 10.1007/BF01456804. MR1511670

Portland State University, P.O. Box 751, Portland, Oregon 97207-0751

E-mail address: gjay@pdx.edu

Department of Mathematical Sciences, Rensselaer Polytechnic Institute, Troy, New YORK 12180

E-mail address: lif@rpi.edu

Department of Aeronautics and Astronautics, Massachusetts Institute of Technology, Cambridge, Massachusetts 02139

E-mail address: cuongng@mit.edu

Department of Aeronautics and Astronautics, Massachusetts Institute of Technology, Cambridge, Massachusetts 02139

E-mail address: peraire@mit.edu 